\title{
AXIAL PROPAGATION OF HELICON WAVES
}

\author{
Max Light, Isaac D. Sudit, Francis F. Chen, \\ and Donald Arnush \\ Electrical Engineering Department
}

PPG- 1541

June, 1995

Submitted to Physics of Plasmas 


\title{
Axial Propagation of Helicon Waves
}

\author{
Max Light, Isaac D. Sudit, Francis. F. Chen, and Donald Arnush \\ Electrical Engineering Department \\ University of California, Los Angeles, CA, 90024-1594
}

Traveling- and standing-wave characteristics of the wave fields have been measured in a helicon discharge using a five-turn, balanced magnetic probe movable along the discharge axis $z$. Helical and planepolarized antennas were used, and the magnitude and direction of the static magnetic field were varied, yielding three primary results. 1) As the density varies along $z$, the local wavelength agrees with the local dispersion relation. 2) Beats in the $z$ variation of the wave intensity do not indicate standing waves but instead are caused by the simultaneous excitation of two radial eigenmodes. Quantitative agreement with theory is obtained. 3) The damping rate of the helicon wave is consistent with theoretical predictions based on collisions alone. 


\section{INTRODUCTION}

Inductively coupled radiofrequency (RF) plasma sources with helicon wave excitation have generated increased interest since Boswell ${ }^{1}$ reported their use to produce high density plasmas efficiently. They have been subsequently used for a variety of applications $^{2-6}$. Radial amplitude profiles of helicon waves in uniform and nonuniform plasmas have been studied extensively by Chen et al..$^{7-9}$ Azimuthal mode content in a high aspect ratio helicon discharge was investigated by Light and Chen, ${ }^{10}$ who found that the $m$ $=+1$ (right-hand polarized) mode was preferentially excited in a variety of antenna and static magnetic field configurations. Though the high ionization efficiency of these helicon discharges is not yet completely understood, Landau and collisional damping 7 , and cyclotron damping ${ }^{11}$ have been suggested as possible mechanisms. Landau damping has also been suggested by Zhu and Boswell ${ }^{12}$ as the mechanism for pumping argon ions to the upper level of the $488 \mathrm{~nm}$ line when the helicon discharge was used to excite an argon laser.

It is important to understand the axial propagation characteristics of the helicon wave before investigating wave-particle interactions in these discharges. For example, the phase velocity $(\omega / k=f \lambda)$ determines the energy of electrons accelerated by the Landau mechanism under the right plasma conditions. Thus, adjusting the parallel wavelength impressed by the antenna, or the excitation frequency, could give some measure of control over the electron energy distribution function. Furthermore, helicon theory predicts that the wavelength depends also on the magnitude (and, for certain antenna types, also direction) of the static magnetic field $\mathbf{B}_{0}$; and this could offer another independent variable parameter.

In this experiment, we investigate the axial propagation of helicon waves in a high aspect ratio $(a / L \ll 1)$ helicon discharge using magnetic probes. Straight (Nagoya Type $\mathrm{III}^{13}$ ) and right-helical (RH) antennas were used in conjunction with varying the magnitude and direction of $\mathbf{B}_{0}$ to study their effect on the standing and traveling wave 
characteristics of the helicon wave. In a separate paper ${ }^{14}$, we have investigated the axial variations of plasma parameters, and the results there support the conclusions drawn here.

\section{EXPERIMENT}

\section{A. The helicon discharge}

The experimental apparatus shown in Fig. 1 has been described elsewhere ${ }^{15}$. The chamber consisted of a quartz tube $1.7 \mathrm{~m}$ in length and $2.5 \mathrm{~cm}$ in diameter. Twelve wire spools were used to create a uniform axial static magnetic field $\mathbf{B}=B_{0} \hat{\mathbf{z}}$ ranging from 0 to 900 Gauss. The antennas investigated were placed on the outside of the glass tube, closer to the end of the tube that leads to the grounded pumping system. The RF current supplied by the $27.12 \mathrm{MHz}$ power supply was fed to the antennas through a double-stub tuning network. A turbomolecular vacuum pump maintained a base pressure of 2-5 x $10^{-5}$ Torr . Argon gas was injected near the midplane of the tube. Typical operating parameters were a fill pressure of $12 \mathrm{mTorr}$, transmitted power of $2.0 \mathrm{~kW}$ with less than $10 \%$ reflection, and an RF pulse length of $150 \mathrm{msec}$.

\section{B. Antennas}

Nagoya Type III and RH antennas were used in this experiment and are shown in Fig. 2. A detailed explanation of the proposed coupling mechanism ${ }^{16}$ for these antennas has been given by Light and Chen ${ }^{10}$. Since the free space wavelength at the frequency of interest for this experiment is much larger than any dimension of the antennas, we can assume that the current reaches all parts of the antenna simultaneously. The current distribution during a half cycle of the RF is shown in Fig. 2. This current will induce an RF magnetic field inside the plasma, which will then induce an RF electric field parallel to $\mathbf{B}_{0}$. This electric field will cause electrons to move along $\mathbf{B}_{\mathbf{0}}$ and set up the space charge distribution needed to reduce the total $E_{\mathrm{z}}$ in the highly conductive plasma to almost zero. On the other hand, the perpendicular electrostatic field set up by the space charge is not canceled because the electrons are confined in the perpendicular direction by the strong 
magnetic field. It is this perpendicular electrostatic electric field which couples efficiently to the perpendicular space charge field of the helicon wave.

The helicity of the $\mathrm{R}$ antenna had the proper sign to couple to the $m=+1$ (righthand circularly polarized) helicon mode pattern. This would set up an electrostatic field which rotates in space, matching the instantaneous pattern of the $m=+1$ mode propagating parallel to $\mathbf{B}_{\mathbf{0}}$, and of the $m=-1$ mode propagating antiparallel to $\mathbf{B}_{\mathbf{0}}$. However, this design does not create the rotation in time that the $m=+1$ mode has. To do this would require phase shifted bifilar antennas, which were not available. Fortunately, excitation of the unwanted $m=-1$ mode in the opposite direction does not occur, for reasons not yet known, as reported earlier ${ }^{10}$. The $\mathrm{N}$ antenna was expected to give a plane polarized wave, created by a linear combination of the +1 and -1 modes; but in practice it excites only the +1 mode in both directions ${ }^{10}$. In order to keep track of the relation between wave propagation and magnetic field direction, the following nomenclature was adopted. We assume that the dominant direction of $\mathbf{k}$ is from the antenna to the midplane of the tube. When the magnetic field points in this direction (to the right in Fig. 1) it will be referred to as a "parallel" (\|) field; when it points towards the pumping port, it will be referred to as an "antiparallel" (\#) field.

\section{Diagnostics}

The first attempt to measure the magnitude of $B_{z}(z)$ was done with an unbalanced, single-turn magnetic probe such as was used previously ${ }^{10}$ for radial profiles. This was made from 0.034 " o.d. semi-rigid coaxial cable, with the center conductor connected to the outer conductor at the end of a loop of about $1 \mathrm{~mm}$ radius. The coax was covered with heat shrink tubing up to the loop. A grounded strip of aluminum foil was wound around the heat shrink tubing in candy-stripe fashion to fit inside a 0.25 " glass tube. For these axial sweeps, the tube was bent into a dog-leg to swing the probe into the plasma. The shaft was fed through a vacuum fitting in the end plate opposite the vacuum end of the chamber and rested on the bottom of the quartz tube. Calibration was carried out by using a single-turn calibration loop driven at the operating frequency if $27.12 \mathrm{MHz}$. The current in this loop was measured with a noninductive 50 -ohm resistor connected to the 
grounded end of the loop. When the magnetic probe was placed at the center of this loop, the measured probe voltage (induced emf) matched the theoretically expected results for the area of the probe and for the $B_{z}$ field generated at the center of a circular hoop with the measured current. However, as the probe was passed axially through the center of the calibration hoop, the probe voltage did not decrease as expected, showing that capacitive pickup affected the probe response. The errors were attributed to insufficient shielding by the semi-rigid coax because of its long length.

A balanced five-turn dog-leg magnetic probe was then constructed (Fig. 3) to reach a plasma radius of $0.4 a$, where $a$ is the tube radius. The outer conductors of two semi-rigid coaxial cables were soldered together with a continuous joint. Five turns of formvar-covered wire were wound around a $1.2 \mathrm{~mm}$ radius and soldered to the center conductors of the semi-rigid cables. The wires between the five turns and center conductors (the dog-leg section) were twisted and shielded with copper tape soldered to the outer conductors of the cables to prevent coupling of unwanted magnetic fields to the probe. The entire assembly was sealed in 1/4" Pyrex tubing except the last few centimeters of the coax cables. These were allowed to protrude out the end of the tube to facilitate connection to a center tapped 1:1 RF transformer via the center conductors. This probe construction gave excellent capacitive signal rejection during calibration, which was carried out in the same manner as for the unbalanced probe. Access to the discharge was also done the same way as for the unbalanced probe.

Stationary wave patterns of $\left|B_{\mathrm{Z}}(z)\right|$ were measured by positioning the probe head about 8 to $10 \mathrm{~cm}$ downstream from the antenna and pulling it back in 1 or $2 \mathrm{~cm}$ increments. Waveforms were recorded on a digital oscilloscope. At each position, the magnitude was recorded as the peak-to-peak value averaged over five shots. The phase of $B_{\mathrm{Z}}(z)$ was measured using two probes in an interferometer arrangement. A single-turn probe was radially inserted into the plasma through a vacuum fitting $24 \mathrm{~cm}$ downstream from the antenna and oriented to measure $B_{\mathrm{Z}}$. The radial position of this probe was kept fixed. The axial probe was juxtaposed to the fixed probe at the start. Both probe signals were fed to separate channels of the digital oscilloscope and overlayed on the same screen. 
The axial probe was then moved downstream from the antenna, and the phase of the two waveforms was observed on the oscilloscope. Axial positions where the two waveforms were in phase and $180^{\circ}$ out of phase were recorded. In the data presented below, the errors were smaller than the size of the data points. That is, the discharge was stable enough that the five shots which were averaged were essentially identical. The small deviations from a smooth curve were not due to noise but were reproducible in the same run. Fig. (4) compares the quality of the data taken with the single-turn unbalanced probe and the five-turn balanced probe, showing the effect of signal feedthrough along the length of the unbalanced probe.

\section{RESULTS}

Figures 5 and 6 show the amplitude of $B_{\mathrm{z}}(z)$ for an RH antenna and a Nagoya III antenna, respectively, for various values of $B_{0}$. The shaded regions represent the front and back rings of the antennas. Several features in the measured field amplitudes are common to both antennas. As the magnitude of $B_{0}$ is increased, a pattern emerges showing definite maxima and minima with the magnitude of $B_{z}(z)$ decreasing away from the antenna. A clear peak at all field magnitudes appear near the front ring of both kinds of antennas. For the case $B_{\mathrm{o}}=0$ (Figs. 5a and 6a), a small peak is located approximately $50 \mathrm{~cm}$ from the center of the antenna, near the location of a grounded flange were the gas is fed into the tube and where a pressure gauge is located. This sensitivity to gas feed location disappears when $B_{\mathrm{o}}$ is large enough for helicon waves to exist. For large values of $B_{0}$, clear minima in the patterns for both antennas appear at $20 \mathrm{~cm}$ and $40 \mathrm{~cm}$ from the center of the antenna, positions that do no correspond to any feature in the vacuum or magnetic coil system. The most significant difference between the standing wave measurements for each antenna is the larger downstream amplitudes measured with the RH antenna.

The "parallel" direction of the magnetic field is conducive to the excitation of the $m=+1$ azimuthal helicon mode when an RH antenna is used ${ }^{10}$. When the field is antiparallel, excitation of the $m=-1$ mode is expected. The effect of field direction on 
wave propagation is illustrated in Figs. $7 \mathrm{a}$ and $7 \mathrm{~b}$. When the axial field is reversed from parallel to antiparallel, the standing wave pattern produced by an RH antenna basically disappears, indicating that the $m=-1$ mode is not excited. This is confirmed by plasma density and optical emission measurements, which show a much weaker plasma when the conditions favor excitation of $m=-1$ instead of $m=+1$. For a Nagoya III antenna, which can excite the $m=+1$ mode in either direction, the downstream wave patterns are slightly different for the two field directions but the magnitudes are comparable.

Interferometric phase shift measurements between two magnetic probes are shown in Fig. 8a for an RH antenna. Though not apparent in this graph, the deviations from a straight line are significant and yield an effective traveling wavelength $\lambda$, calculated from the slope $d \varphi / d x$ using the equation:

$$
\lambda=360(d \varphi / d x)^{-1}
$$

Fig. $8 \mathrm{~b}$ shows the traveling wavelengths as a function of axial position and magnetic field for an RH antenna. Near the antenna, the wavelengths are approximately $12 \mathrm{~cm}$ for all magnetic fields. In all cases, a similar behavior is observed: the wavelengths initially decrease, reaching a minimum, and subsequently increase. The minimum wavelength occurs farther from the antenna as the axial magnetic field increases in magnitude. We shall show that this behavior is related to the axial density profiles $n(z)$ obtained at each field. For the Nagoya III antenna, the corresponding phase shift measurements and derived wavelengths are presented in Figs. 9a and 9b. In contrast to the RH antenna, no obvious trend is detected as $B_{\mathrm{o}}$ is varied. The wavelengths seem to be longer than for the helical antenna and decrease with magnetic field magnitude, reaching a constant value for $B_{\mathrm{o}} \geq 800 \mathrm{G}$.

\section{ANALYSIS}

\section{A. Standing wave measurements}

The standing wave patterns that emerge at high magnetic fields, shown in Figs. 5 and 6, exhibit a decaying amplitude and non-zero minima. The wave amplitude decreases 
to such a small amplitude at the end of the tube that these patterns cannot be caused by reflections from the grounded end-flange. One possible explanation for the origin of the "standing waves", and the one we have decided to investigate, is the simultaneous excitation by the antenna of two different helicon modes. These modes can differ in azimuthal mode number $m$, or in radial mode number $n$. We shall find that the first two radial modes, $n=1$ and 2 (for $m=+1$ ), will give reasonable agreement with the observed periodicity of the standing wave pattern.

Consider two undamped eigenmodes with $B_{\mathrm{z}}$ amplitudes $B_{1}(r, z)$ and $B_{2}(r, z)$ and wave numbers $k_{1}(z)$ and $k_{2}(z)$, respectively. The total field $B_{\mathrm{t}}(r, z, t)$ is given, in a WKB sense, by

$$
\left.B_{t}=B_{1} \sin \vec{z}_{z_{0}}\left(z^{\prime}\right) d z^{\prime}+\omega t \mathbf{K}_{2} \sin \vec{z}_{z_{0}}\left(z^{\prime}\right) d z^{\prime}+\omega t\right)
$$

where $z_{O}$ is the axial position at which the modes are in phase. The peak-to-peak amplitude of $B_{\mathrm{t}}$ can be found straightforwardly by setting the time derivative of $B_{\mathrm{t}}$ to zero. The result is:

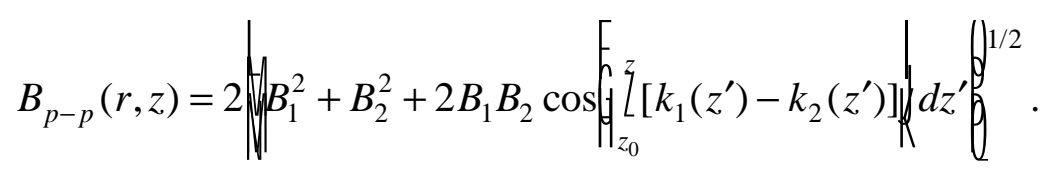

The local maxima and minima of this expression occur where the cosine term is equal to \pm 1 ; these positions depend only on the wavenumbers of the modes and are independent of their relative amplitudes. In the absence of damping, the ratio of the maxima to the minima of the standing wave pattern is equal to.

$$
\frac{B_{1}(r)+B_{2}(r)}{B_{1}(r)-B_{2}(r)}
$$

Since our previous measurements ${ }^{10}$ have shown that the $m=+1$ mode is the only azimuthal mode excited by our antennas, we choose the first two radial modes for $m=+1$ as likely candidates for $B_{1}$ and $B_{2}$. The local wavenumbers $k_{1}(z)$ and $k_{2}(z)$ are calculated 
from the helicon dispersion relation for a nonuniform plasma ${ }^{8}$, using the measured radial density profile $n_{0}(r)$. The differential equation for the radial component $B_{\mathrm{r}}$ is given by ${ }^{9}$

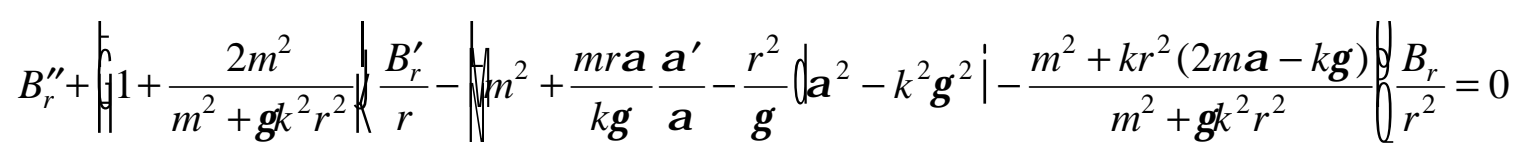

where the prime indicates a radial derivative and

$$
\alpha(r, z)=\frac{\omega}{k(z)} \frac{e \mu_{0}}{B_{0}} n_{0}(r, z), \quad \gamma=1-(\omega / c k)^{2}
$$

Eq.(6) can be considered the local dispersion relation at each $(r, z)$. At each $z$, the radial dependence is accounted for by numerically integrating Eq. (5) using the measured peak values $n_{0}(z)^{14}$ but assuming that the radial profile $n_{0}(r)$, measured ${ }^{10}$ at $z=24 \mathrm{~cm}$, does not change with $z$. The first two radial modes satisfying the boundary conditions ${ }^{9} B_{r}(a)=0$ and $B_{r}^{\prime}(0)=0$ give the values of $k_{1}(z)$ and $k_{2}(z)$.

A small error is incurred in this procedure because the absolute densities $n_{0}(z)$ measured in Ref. 14 were obtained at an argon fill pressure of 15 mTorr, while the $B_{\mathrm{z}}(z)$ data presented here were obtained at 12 mTorr. With this proviso, the calculated wavelengths for the first two $m=+1$ radial modes $(n=1,2)$ for the corresponding axial density profiles for RH and Nagoya III antennas are shown in Figs.10a and 10b, respectively.

Since in practice the waves are damped, we use the minima in Fig. 5e as a baseline and compute the ratio $\left|B_{2}(n=2) / B_{1}(n=1)\right|$ from Eq. (4) for the case of the RH antenna. This ratio is approximately 5; the exact number does not affect the following results significantly. Using this value, the simulated standing wave pattern calculated from Eq. (2) is compared with the measured pattern in Fig. 11a. The curves have been shifted so that the first minima coincide; the in-phase position is then $-2 \mathrm{~cm}$. We see that the spacing of the first two minima is in reasonable agreement with the observed pattern; beyond 40 $\mathrm{cm}$, however, other modes appear which are not accounted for in this treatment. Figure 
$11 \mathrm{~b}$ presents the corresponding results for a Nagoya III antenna. For this case, the ratio $\left|B_{2}(n=2) / B_{1}(n=1)\right|$ is taken to be 3 . Again reasonable agreement is obtained near the antenna, though the data are indistinct because the modes are not as well excited as by the RH antenna. A similar exercise assuming the $m=+3$ azimuthal mode with $n=1$ did not agree with the data as well. Even values of $m$ are not excited with our antenna geometries. Thus, we can explain the observed beat pattern with eigenmodes which exactly satisfy the helicon dispersion relation for a nonuniform plasma.

That the $\mathrm{m}=+1, \mathrm{n}=1$ and 2 modes should be the dominant ones observed can be shown to be consistent with the $k$-spectrum of the vacuum fields generated by the antennas. We have computed the Fourier spectra of the RH and Nagoya III antennas for the exact geometries used, but neglecting the finite width of the conductors. Reflections from the ends of the cylinder are suppressed. The results are shown in Fig. 12, together with the $k$ values calculated from the measured densities and density profiles. It is seen that only the $(1,1)$ and $(1,2)$ modes occur at wavelengths for which the fields of our antennas have a large Fourier amplitude, while the $(3,1)$ mode falls at an antenna minimum.

\section{B. Traveling wave measurements}

We have also attempted to explain the wavelength variation found in the phase measurements, as shown in Fig. 8. In the local dispersion relation, Eq. (6), the total wavenumber $\alpha$ depends only on the tube radius, radial density profile, and mode number. One would then expect $k(z)$ to vary locally with the density $n_{0}(z)$, and therefore $\lambda(z)$ to have a dip where the density has a peak, as shown in Fig. 10. To check this quantitatively, we must consider that there are two radial modes involved. Since the $n=1$ radial mode has much larger amplitude than the $n=2$ mode, we could, in principle, neglect the latter mode in computing the observed wavelength. However, there was no need to do this, since the ratio of amplitudes is known from (A), and we can take both modes into account. The procedure used in the calculation mirrored that used in taking the data. The modes' wavenumbers were computed from Eq. (5) are used in Eq. (2), as before. Starting at the same location where both modes were found to be in phase in (A), a plot of $B_{\mathrm{t}}$ as a 
function of time was generated. The position $z$ was then varied until a new $B_{\mathrm{t}}$ is obtained that was $180^{\circ}$ out of phase. This procedure was repeated for increasing $z$ to generate a series of points differing in phase by $180^{\circ}$ from one to the next. At each $z$, the locally measured density is used in solving Eq. (5). From these phase-vs.-position data we then proceed to calculate wavelengths using Eq. (1). These generated wavelengths are compared with the measured ones in Fig. 13a for the RH antenna and in Fig. 13b for the Nagoya III antenna. Since the data were taken in different runs, the wave measurements were made at 12 mTorr, slightly lower than the 15 mTorr at which the densities were measured. For the RH antenna, the curves agree qualitatively in shape but are off about $50 \%$ in magnitude. The dashed lines show the effect of varying the density used in the calculation by a factor of 2 in either direction. Unfortunately, the best agreement is for the density varying in the opposite direction from what is expected from the pressure difference. For the Nagoya III antenna, the wavelengths again agree within $50 \%$, but the $z$-dependence is not clear.

\section{Damping measurements}

Finally, we consider the damping rate of the waves, which is superimposed on the beating phenomenon. The damping decrement, $k_{\mathrm{i}} \equiv \operatorname{Im}(k)$, has been given by $\mathrm{Chen}^{7}$ for a uniform plasma:

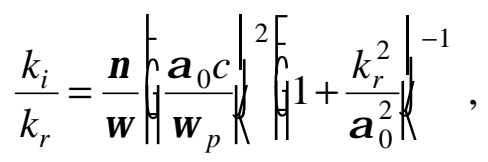

where $k_{\mathrm{r}} \equiv \operatorname{Re}(\mathrm{k})$ and $\alpha_{0}$ is the value of $\alpha$ computed without damping. For this calculation, we consider only the dominant $(1,1)$ mode and have replaced the radial density profile with an equivalent square profile containing the same number of particles. The parameter $\alpha$ is then given by Eq. (6) (without the r-dependence) and is proportional to $1 / k_{\mathrm{r}}$. Using this in the boundary condition [Ref. 7, Eq. (35)] for a uniform plasma yields the local value of $k_{\mathrm{r}}$ taking into account the measured profile $n_{0}(z)$ (Fig. 10). To calculate 
$k_{\mathrm{i}}$, we must also evaluate the effective collision frequency $v$, which will depend on both the $T_{\mathrm{e}}(z)$ and $n_{0}(z)$ profiles.

We have considered four dissipative processes. For electron collisions with neutral atoms, we use the value of $\langle\sigma v\rangle$ computed with the measured local temperature. The neutral density was assumed to be depleted by a factor of four, as found previously ${ }^{14}$. For electron-ion collisions, the dominant mechanism, we used the classical Spitzer resistivity at the local temperature. Landau damping can arise from resonant electrons in the tail of the thermal distribution or from a population of accelerated "beam" electrons. At the 2-5 eV temperatures here, Landau damping from the thermals is entirely negligible. We do not know how to estimate the number of "beam" electrons, since we have never observed any at densities above $10^{13} \mathrm{~cm}^{-3}$, but this mechanism was not needed to explain the data, and we have neglected it. Finally, we have considered damping caused by the finite lifetime of the particles. When an electron oscillating in the wave leaves the discharge and is replaced by a newly ionized one, memory of the oscillation is lost, and the wave loses energy proportional to the rate at which the particles leave the discharge. Though important for other types of waves, lifetime damping is negligible for helicon discharges for two reasons. First, as shown by $\mathrm{Chen}^{7}$, the dominant part of the wave energy is in the oscillating magnetic field, and this is not affected by the replacement of an oscillating electron by a newly ionized one, since the currents generating the magnetic field are carried by drifts. The electric field, though large, carries energy lower than this by a factor $(\omega / k c)^{2}$. The kinetic energy of the ions is negligible because they can hardly move at the wave frequency. The kinetic energy of the electrons is not small, but the part that is associated with the wave is only the $\mathbf{E} \times \mathbf{B}$ small motion, which carries a minute amount of energy. Secondly, in a long discharge, particles are lost primarily in the radial direction ${ }^{14}$. The amount of wave energy carried out by particles striking the wall must be small, since the boundary condition requires the oscillating electron velocity to vanish there.

For these reasons, we have used the sum of electron-ion and electron-neutral collision frequencies for $v$ in Eq. (7) to evaluate $k_{\mathrm{i}}(z)$. The wave amplitude then falls as 




This curve is shown in Fig. 14 in comparison with the data on $\left|B_{z}\right|$. The slope is in reasonable agreement with the observed damping rate of the wave. Since the neutral

density is not known exactly becauseof the ion-pumping effect ${ }^{14}$, curves for half and twice the assumed neutral density are also shown; the differences are small because electron-ion collisions are dominant.

\section{CONCLUSIONS}

We have measured the wave magnitudes and phases along the axis of a high aspect ratio helicon discharge. The data are in agreement with calculations based on measured density and temperature profiles without the assumption of non-classical processes such as the presence of fast electron tails. The wave damping rate and the variation of local wavelength are in reasonable agreement with the helicon wave dispersion relation. An unexpected result is that the standing-wave pattern often seen in such discharges is not caused by reflections from the end boundaries but by the simultaneous excitation of two helicon modes by the antenna. The two modes most strongly excited by the antenna configurations used are found to have wavelengths whose beat length agrees with the observed standing-wave pattern.

\section{ACKNOWLEDGMENTS}

This work was supported by the National Science Foundation, Grant No. ECS9400849; the Semiconductor Research Corp., Project 93-MJ-529; the Wisconsin Engineering Research Center for Plasma-Aided Manufacturing, and the Plasma Physics Research Institute of the Lawrence Livermore Laboratory. 


\section{FIGURE CAPTIONS}

Fig. 1. Schematic of the apparatus. The tube is $4.7 \mathrm{~cm}$ in diameter and $165 \mathrm{~cm}$ long. The argon pressure was $12 \mathrm{mT}$ Torr, and the RF input power was kept at $2.0 \mathrm{~kW}$. The magnetic field coils produce a uniform axial field of up to $1.2 \mathrm{kG}$.

Fig. 2. Nagoya III antenna (a) and right-helical antenna (b) used to excite helicon waves and to produce the plasma. The small arrows show the RF current at one phase; the large arrows indicate the electrostatic field due to the axial displacement of the electrons.

Fig. 3. Diagram of the magnetic probe designed to reject capacitive pickup and detect the $z$ component, $B_{\mathrm{z}}$, of the helicon wave magnetic field. The center of the five-turn, $1.2 \mathrm{~mm}$ diam. coil is located at $r / a=0.4$, near the maximum of the radial profile of $\left|B_{\mathrm{Z}}\right|$.

Fig. 4. Comparison between the balanced (-_-) and unbalanced (-O-) $\left|B_{\mathrm{z}}\right|$ signals for an $800-\mathrm{G} \mathrm{B} \| \mathbf{k}$ argon discharge, showing superiority of the balanced probe in capacitive pickup rejection.

Fig. 5. Axial variation of $\left|B_{\mathrm{Z}}\right|$ at various magnetic fields $B_{\mathrm{o}}$ from (a) $0 \mathrm{G}$ to (f) $900 \mathrm{G}$, for a right-helical antenna with $\mathbf{B} \| \mathbf{k}$. The shaded areas correspond to the front and back rings of the antenna. Only at $900 \mathrm{G}$ was the probe swept through the entire antenna region.

Fig. 6. Same as Fig. 5, but for a Nagoya III antenna.

Fig. 7. Change in the axial profiles of $\left|B_{Z}\right|$ as the direction of a 900-G magnetic field is reversed. (a) Right-helical antenna; (b) Nagoya III antenna.

Fig.8. (a) Phase of traveling helicon waves vs. $z$ for a right-helical antenna with $\mathbf{B} \| \mathbf{k}$, for various magnetic fields. (b) Wavelengths derived from the slope of the curves in (a).

Fig. 9. Same as Fig. 8, but for a Nagoya III antenna. 
Fig.10. Measured plasma density and calculated wavelengths of the first two radial $m=+1$ modes for 15-mTorr, 800-G, B || $\mathbf{k}$ argon plasmas excited by (a) a right-helical and (b) a Nagoya III antenna.

Fig.11. Comparison of calculated (----) standing-wave patterns with measurements ( $-\mathrm{O}-$ ) for $800-\mathrm{G}, \mathbf{B}|| \mathbf{k}$ plasmas produced by (a) an $\mathrm{RH}$ antenna and (b) a Nagoya III antenna.

Fig.12. (a) Computed $k$-spectrum of a half-wavelength $R H$ antenna of length $L$ for the first few azimuthal modes. Even modes are not excited. The arrows show the computed values of $k$ for the $(m, n)=(1,1),(1,2)$, and $(3,1)$ modes in a nonuniform plasma. (b) Same for a half-wavelength Nagoya III antenna.

Fig.13. Measured (——) and calculated ( $~-)$ traveling-wave wavelengths for an 800G, B $\| \mathbf{k}$ plasma produced by (a) a right-helical antenna and (b) a Nagoya III antenna. The wavelengths were measured in a 12-mTorr argon plasma, while the calculations used densities measured in a 15-mTorr plasma. The upper and lower dashed lines were calculated with half and twice the measured densities, respectively.

Fig. 14. Computed wave damping (lines) compared with measured wave amplitude along $z$. The dashed lines are for half and twice the expected neutral density, showing that electron-ion collisions are dominant. 


\section{REFERENCES}

1Boswell, R. W., Plasma Phys. and Controlled Fusion 26, 1147 (1984).

2Perry, A. J., Vender, D., and Boswell, R. W., J. Vac. Sci. Technol. B 9, 310 (1991).

${ }^{3}$ Nakano, T., Gottscho, R. A., Sadeghi, N., Trevor, D. J., Boswell, R. W., Perry, A. J., Lee, T. C., Giapis, K. P., and Margot, J., "Oyo Buturi”, Japan Soc. of Appl. Phys. 61, 711 (1992).

${ }^{4}$ Chen, F. F., "Helicon Plasma Sources", in High Density Plasma Sources, ed. by Oleg A. Popov (Noyes Publications, Park Ridge, N.J.), to be published (1995).

${ }^{5}$ Chen, F. F., Physica Scripta T30, 14 (1990).

${ }^{6}$ Lowenhardt, P. K., Blackwell, B. D., Boswell, R. W., Conway, G. D., and Hamberger, S. M, Phys. Rev. Lett. 67, 2792 (1991).

${ }^{7}$ Chen, F. F., Plasma Phys. and Controlled Fusion 33, 339 (1991).

${ }^{8}$ Chen, F. F., Hsieh, M. J., and Light, M., Plasma Sources Sci. Technol. 3, 49 (1994).

${ }^{9}$ Sudit, I.D. and Chen, F.F., Plasma Sources Sci. Technol. 3, 602 (1994).

10Light, M., and Chen, F. F., Physics of Plasmas 2, 1084 (1995).

${ }^{11}$ Harvey, B. M., and Lashmore-Davies, C. N., Phys. Fluids B 5, 3864 (1993).

12Zhu, P., and Boswell, R. W., Phys. Rev. Lett. 63, 2805 (1989).

${ }^{13}$ Okamura, S., Adati, K., Aoki, T., Baker, D. R., Fujita, H., Garner, H. R., Hattori, K., Hidekuma, S., Kawamoto, T., Kumazawa, R., Okubo, Y., and Sato, T., Nuclear Fusion 26, 1491 (1986).

${ }^{14}$ Sudit, I. D. and Chen, F. F., "Remote Source Characteristics of Helicon Discharges", UCLA PPG-1534 (1995), submitted to Plasma Sources Sci. Technol.

${ }^{15}$ Chen, F.F. and Chevalier, G., J. Vac. Sci. Technol. A 10, 1389 (1992).

${ }^{16}$ Chen, F., F., TRW Report Task II-3552 (1981, unpublished). 


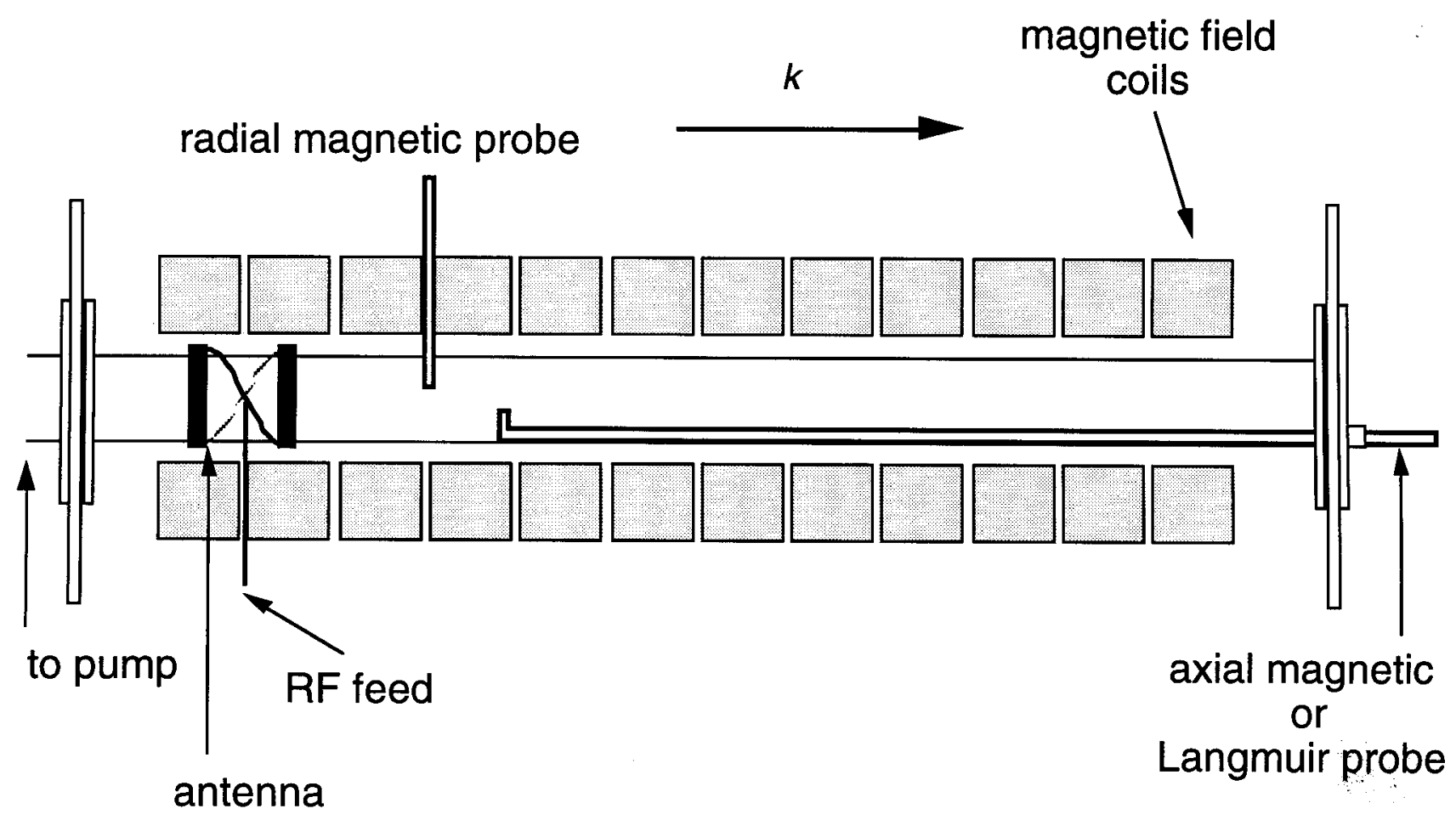

Fig. 1 


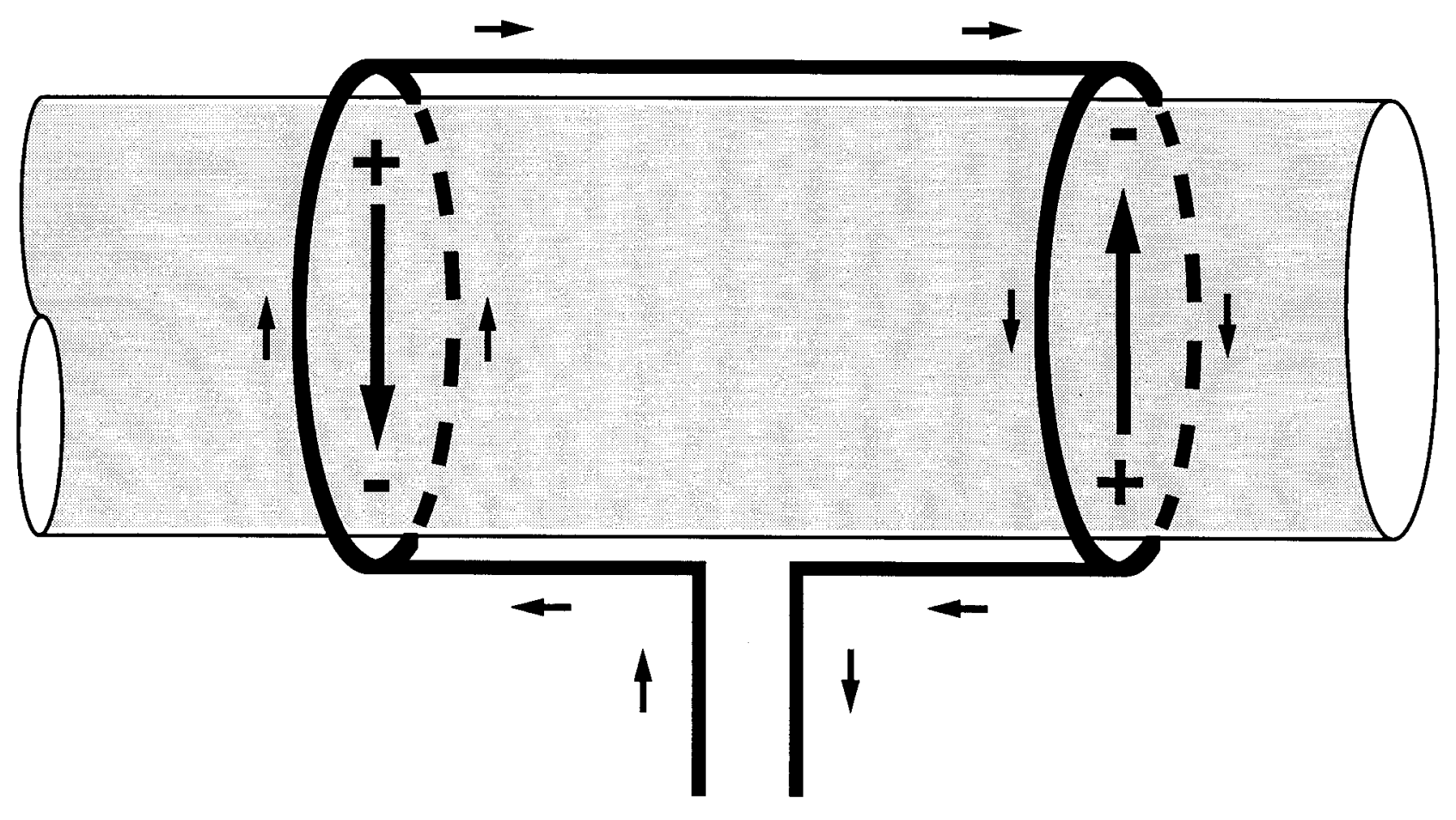

Fig. 2a 


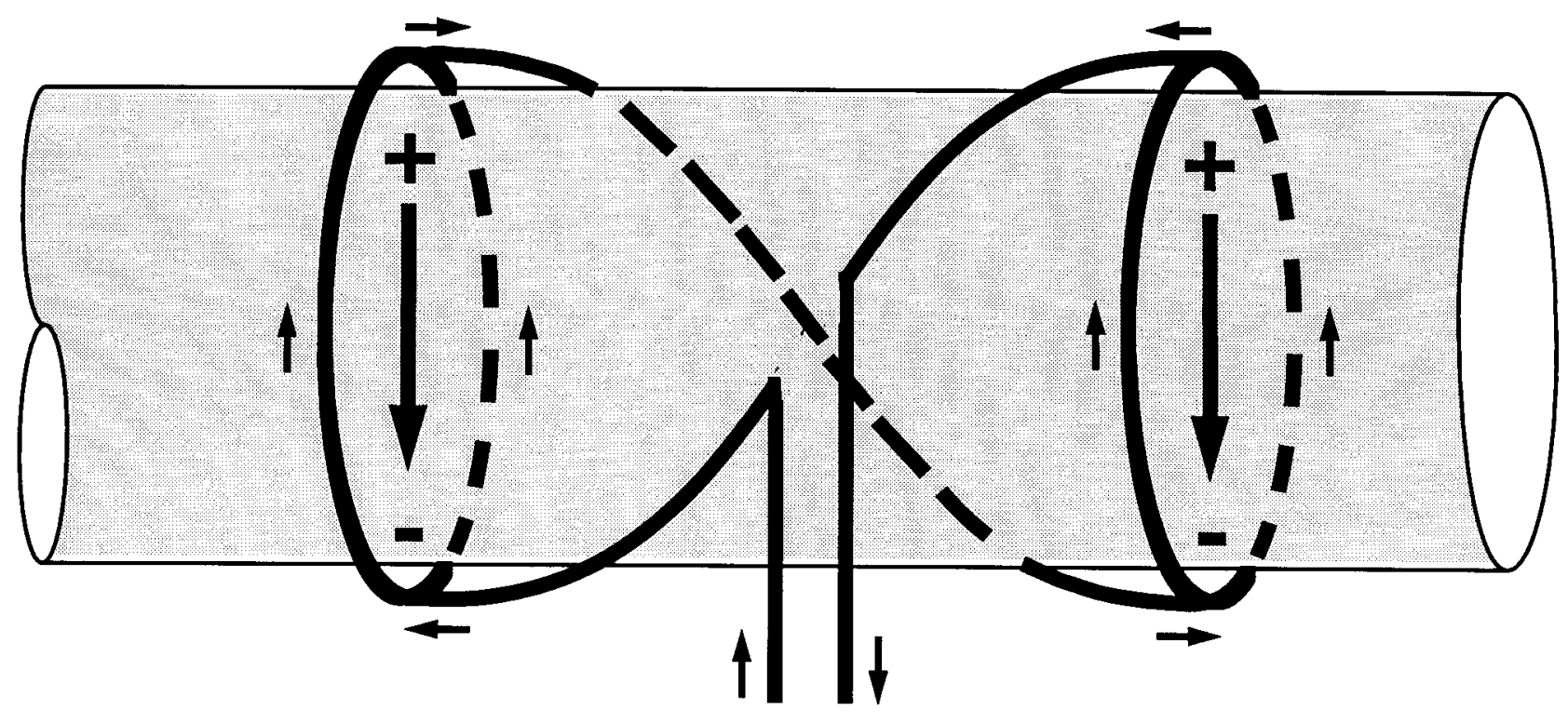

Fig. 2b 


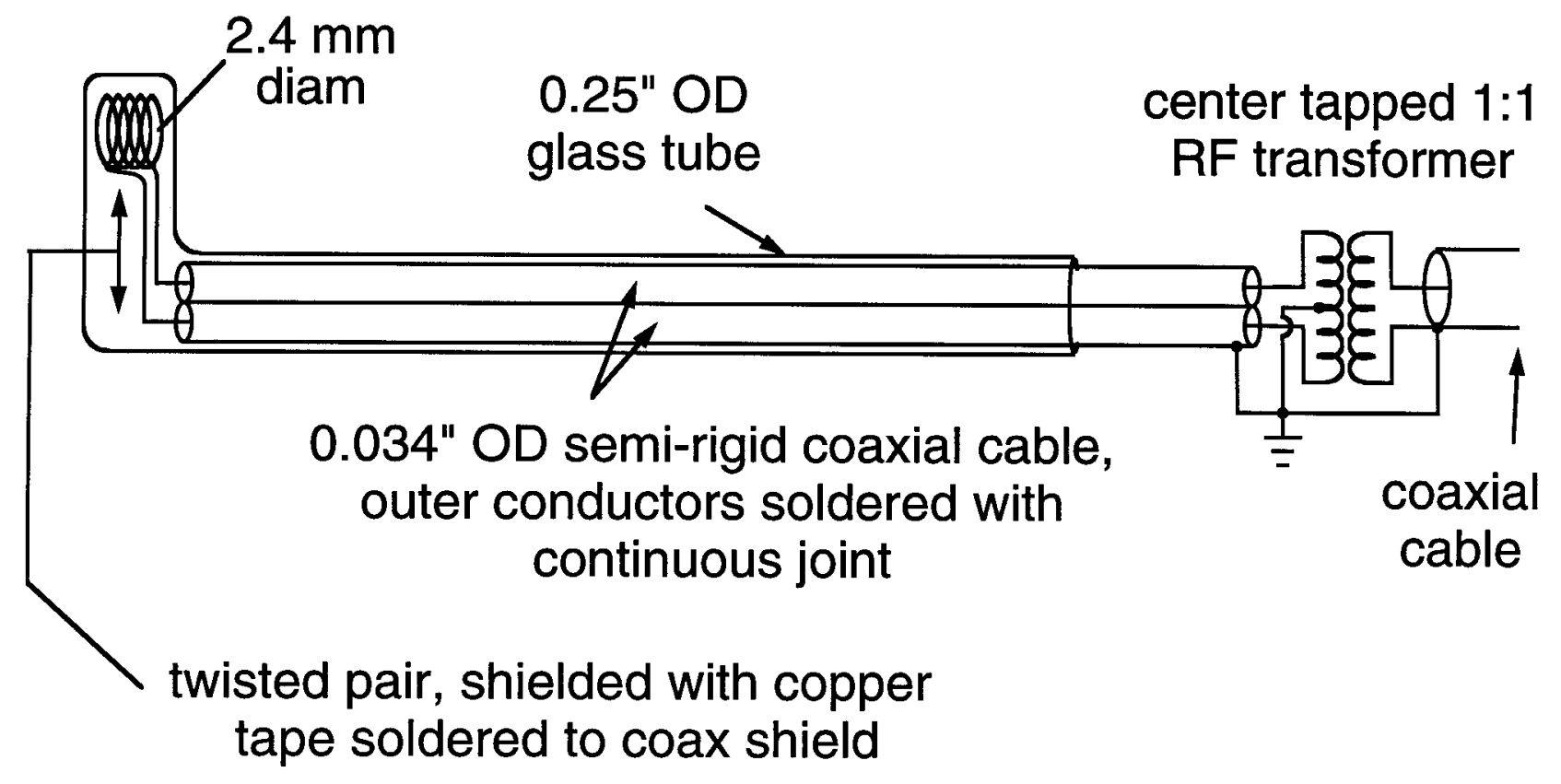

Fig. 3 


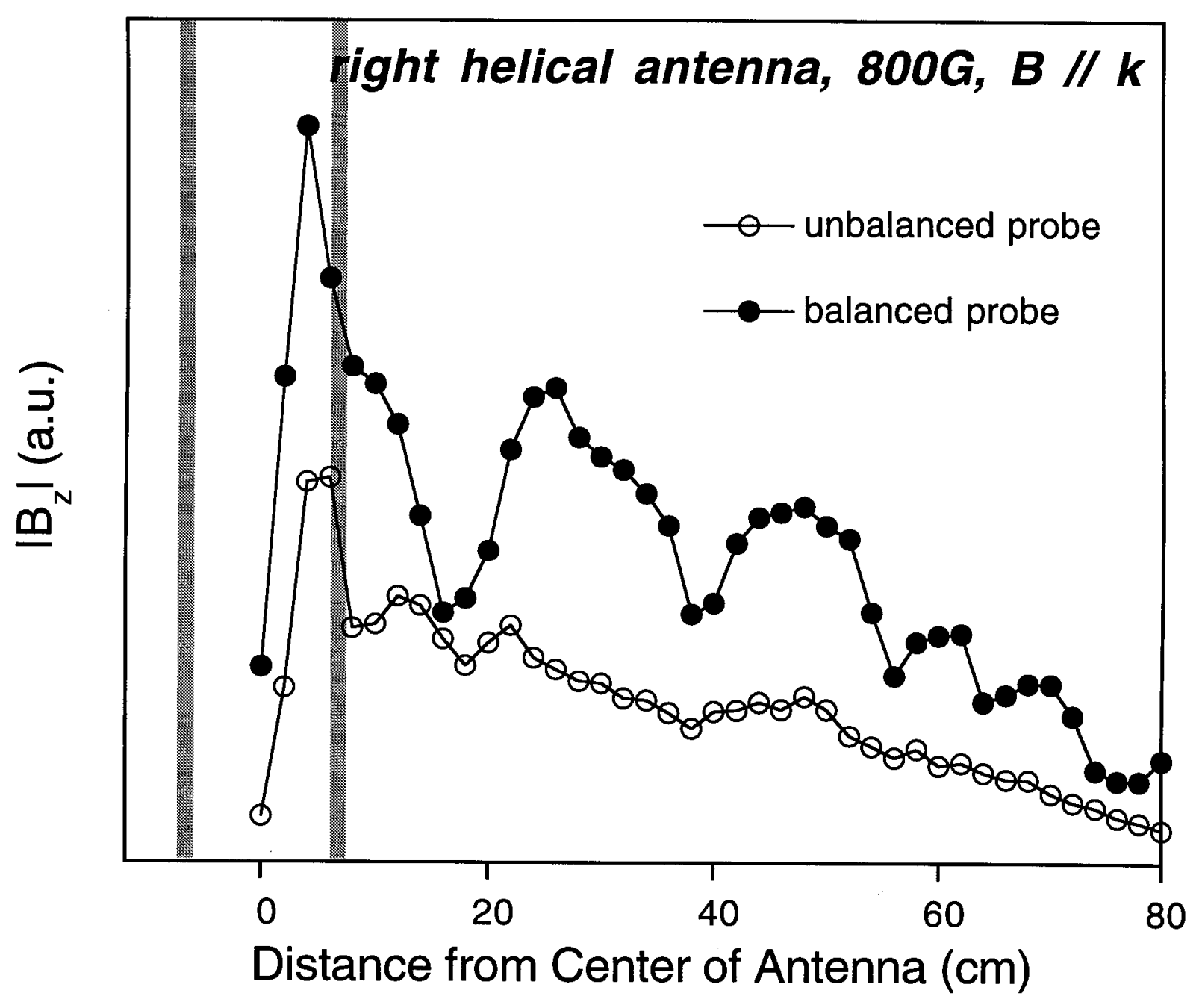

Fig. 4 


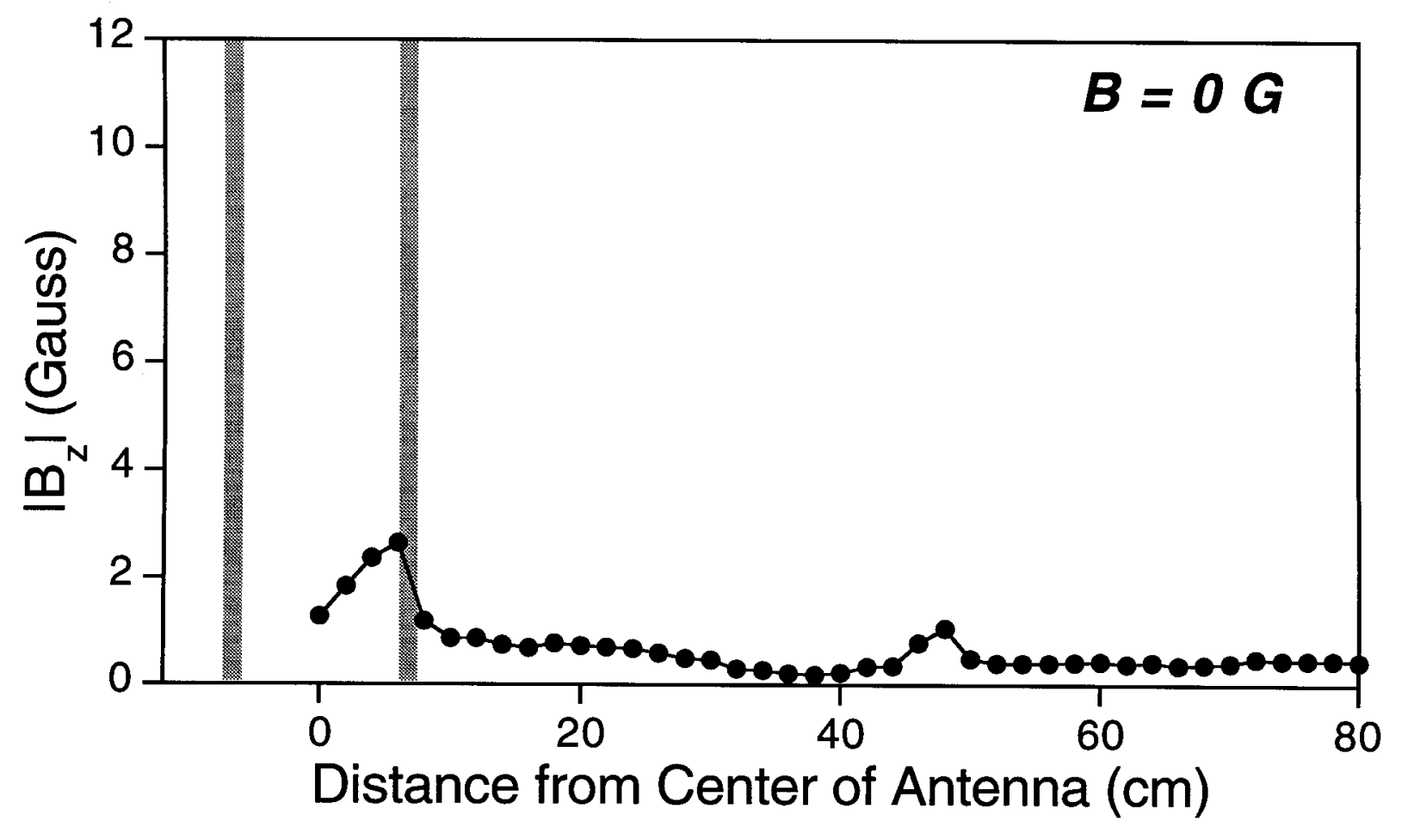

Fig. 5a 


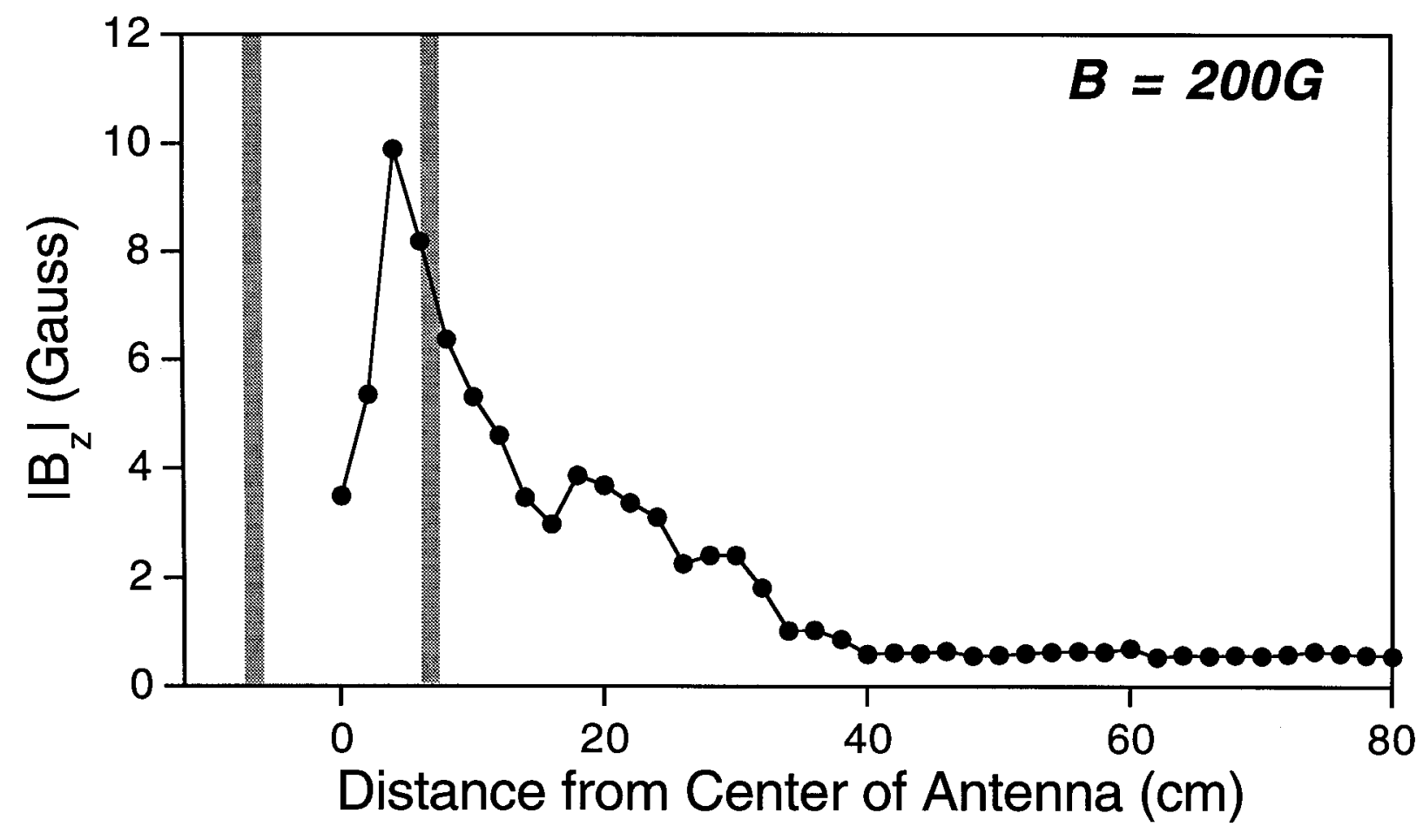

Fig. 5b 




Fig. $5 \mathrm{c}$ 


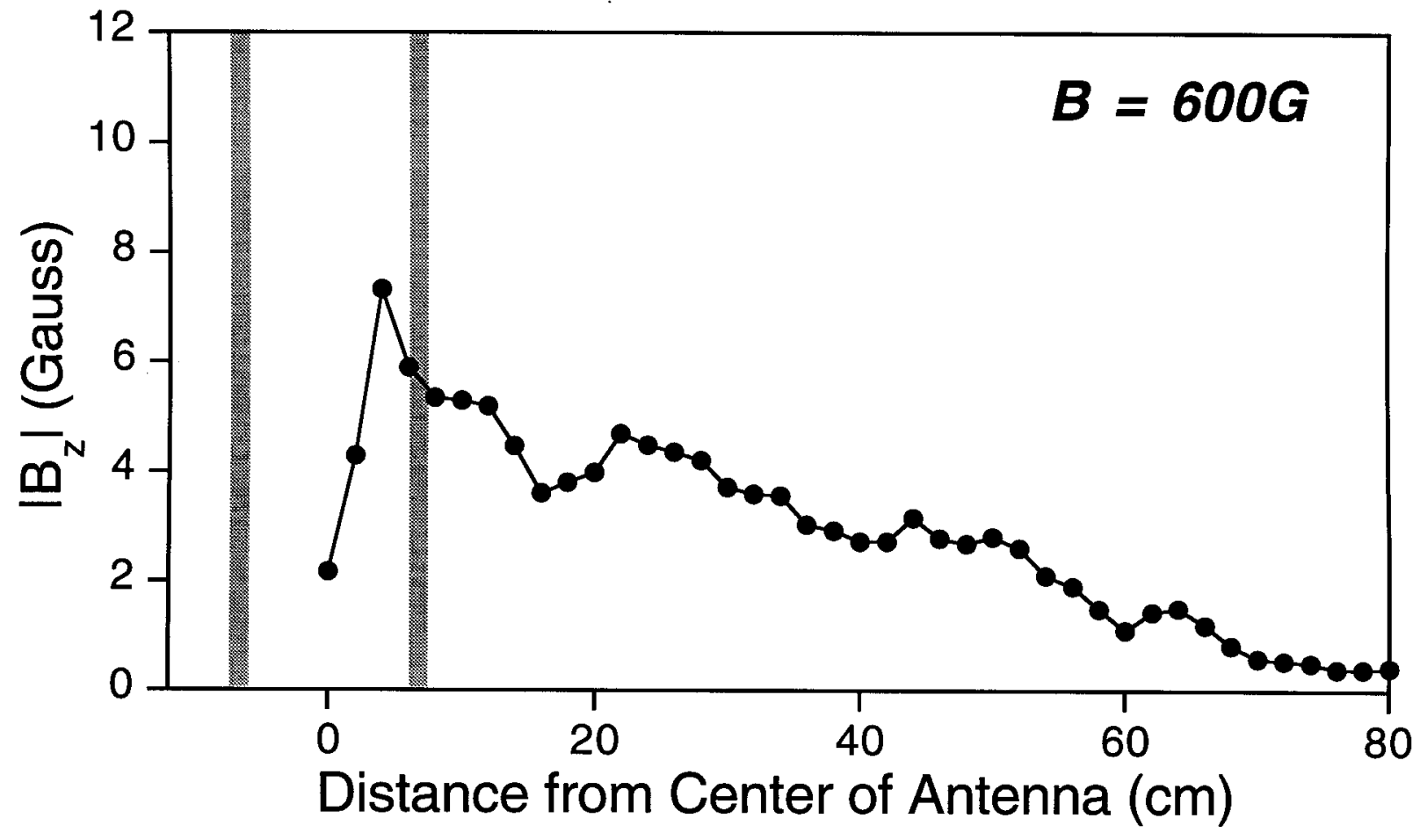

Fig. 5d 


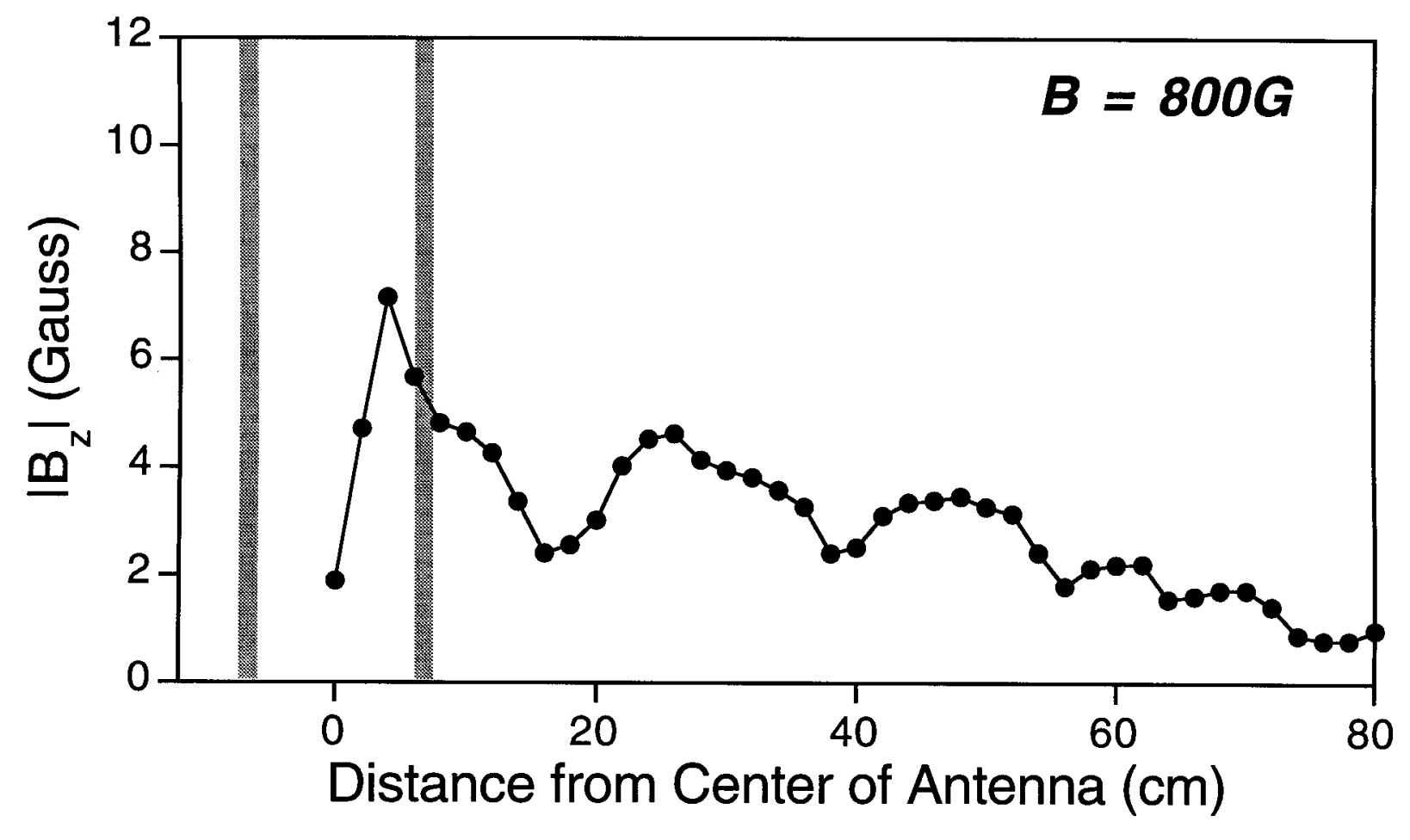

Fig. 5e 


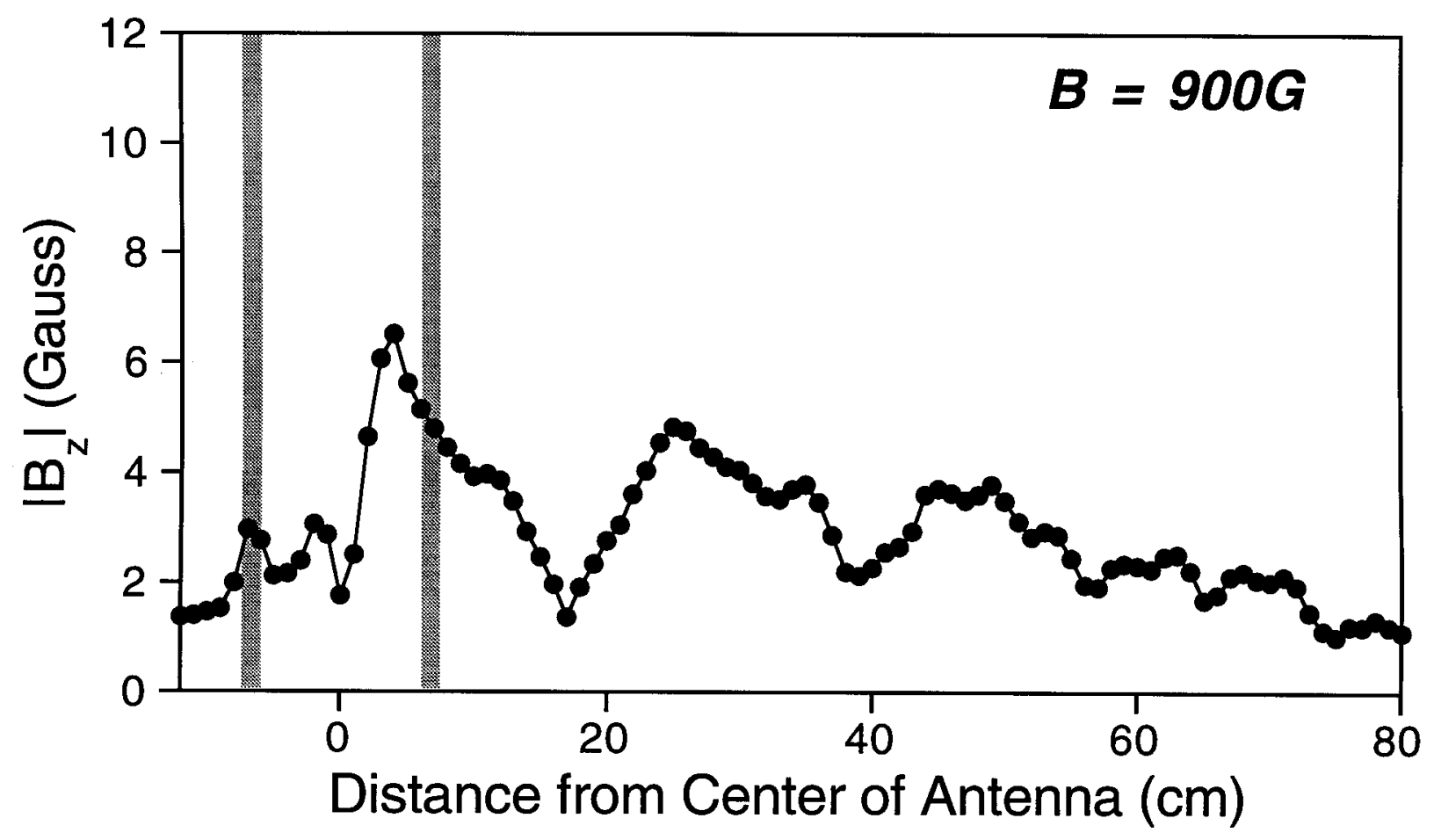

Fig. $5 f$ 


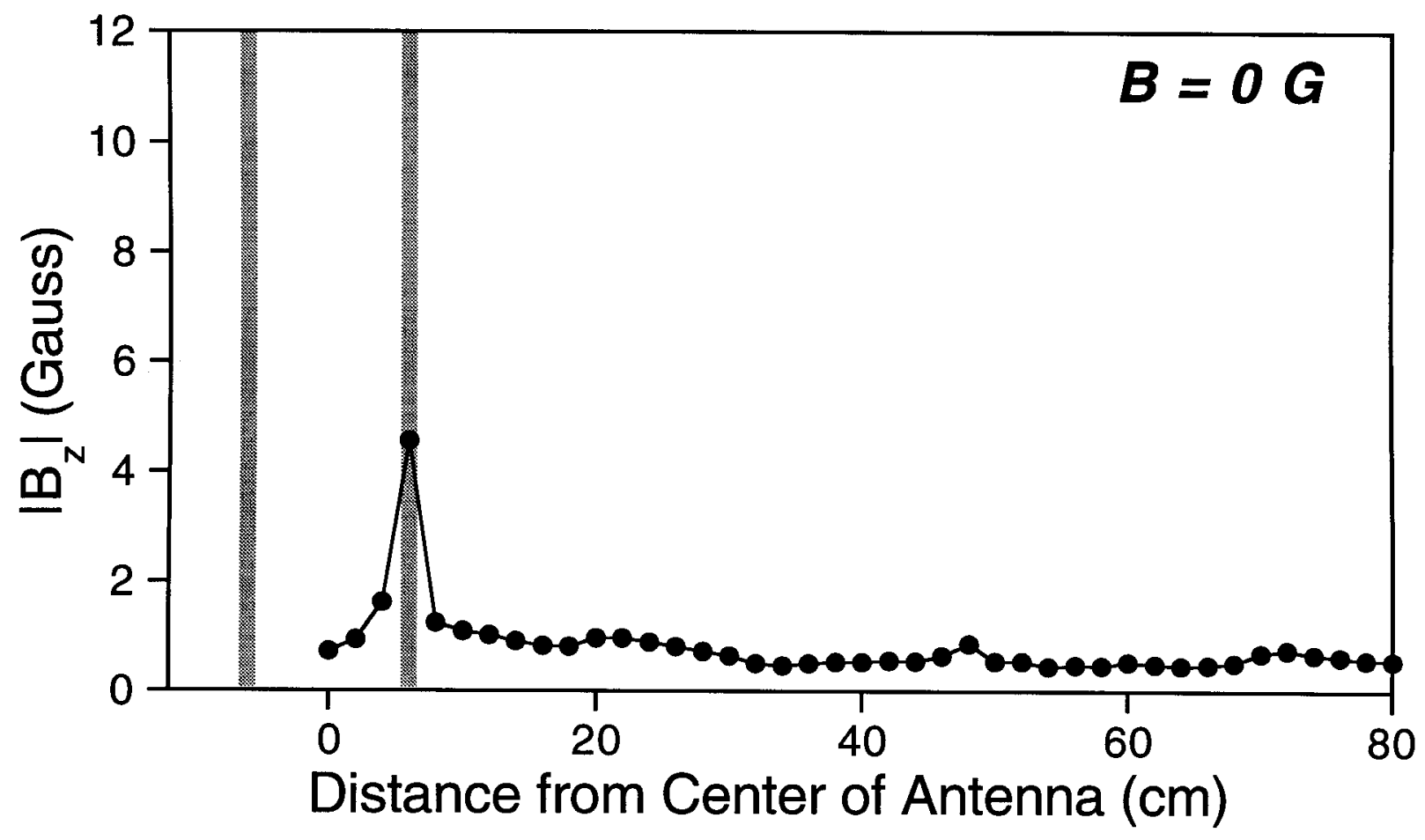

Fig. 6a 


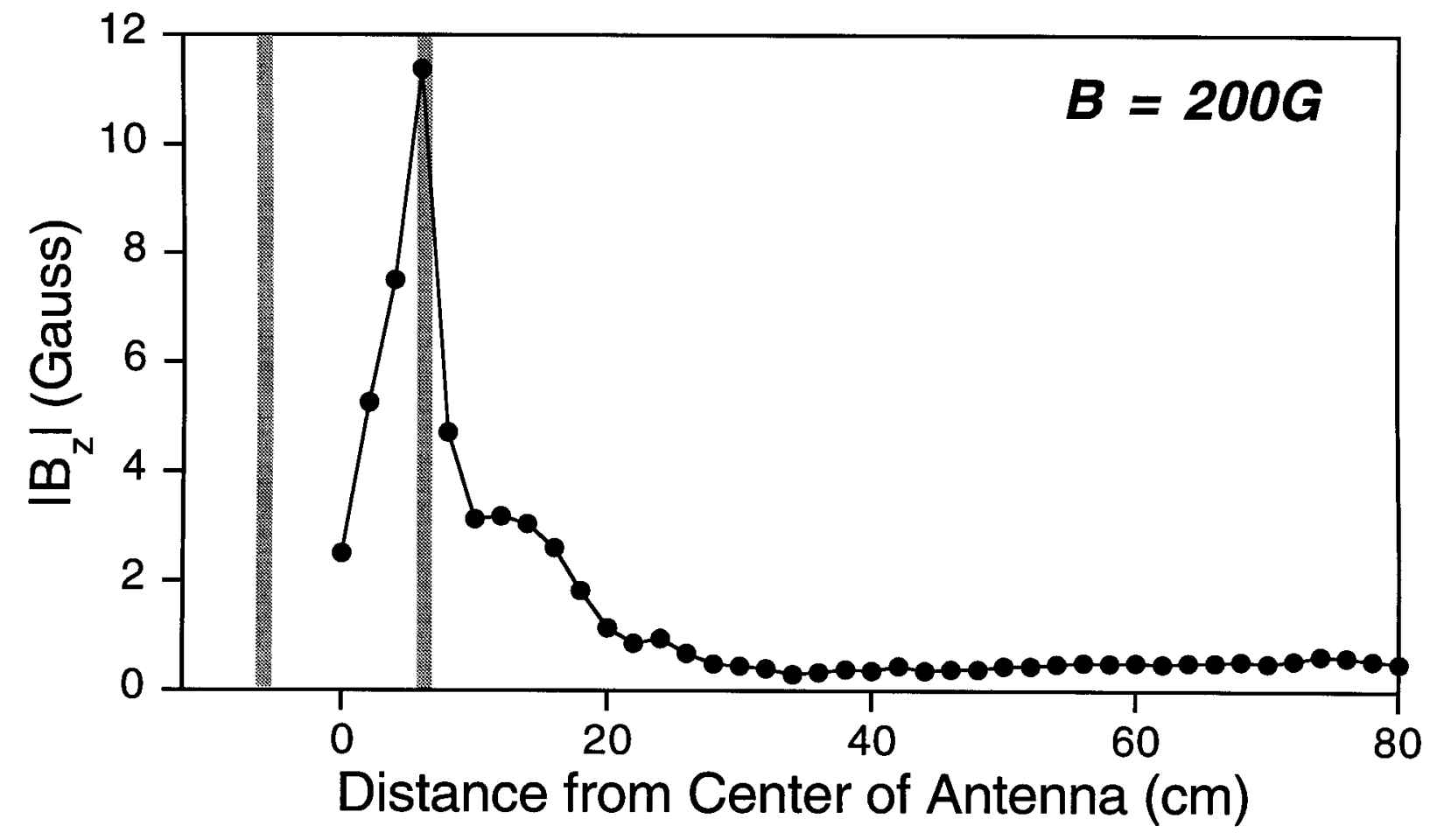

Fig. $6 b$ 


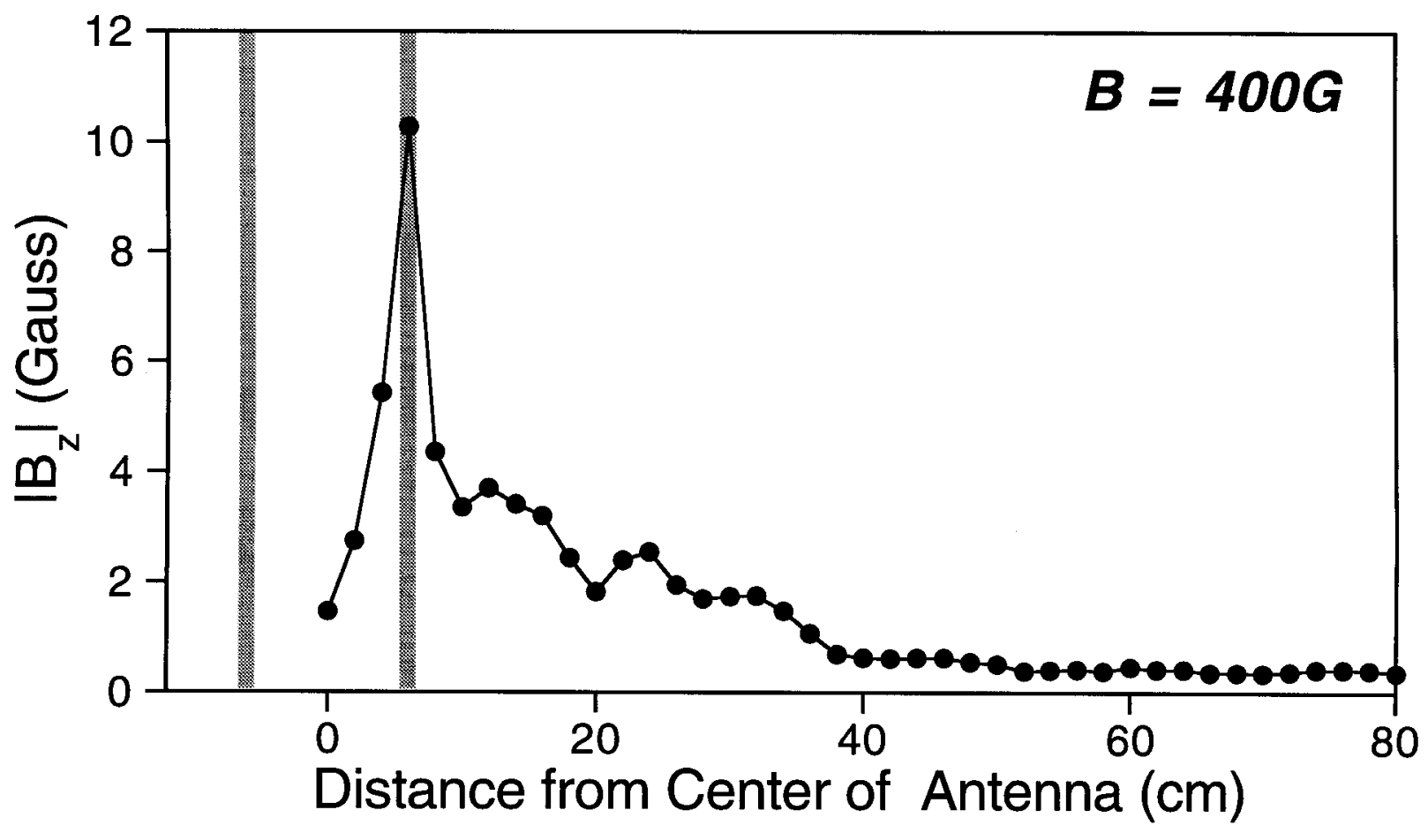

Fig. 6c 


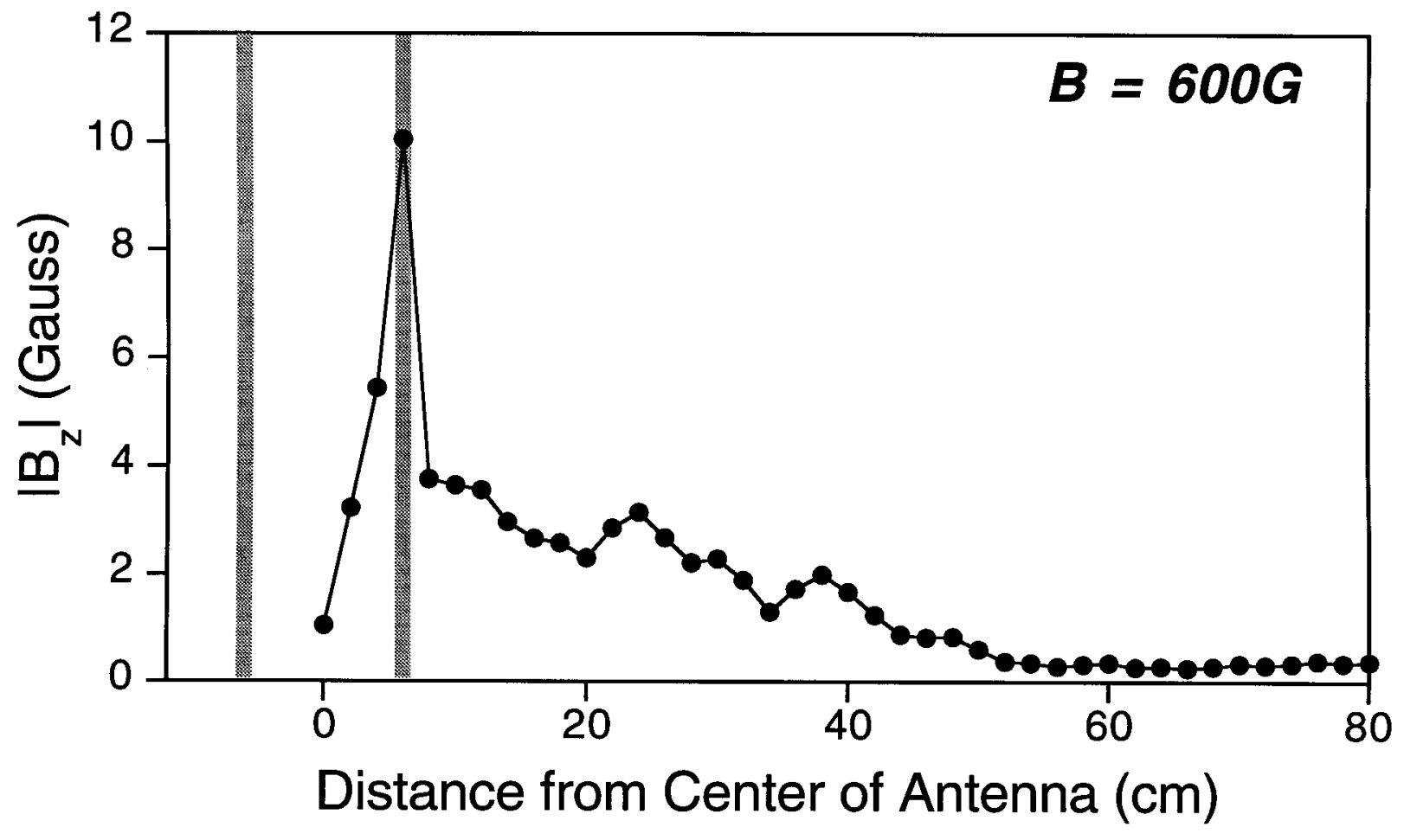

Fig. 6d 


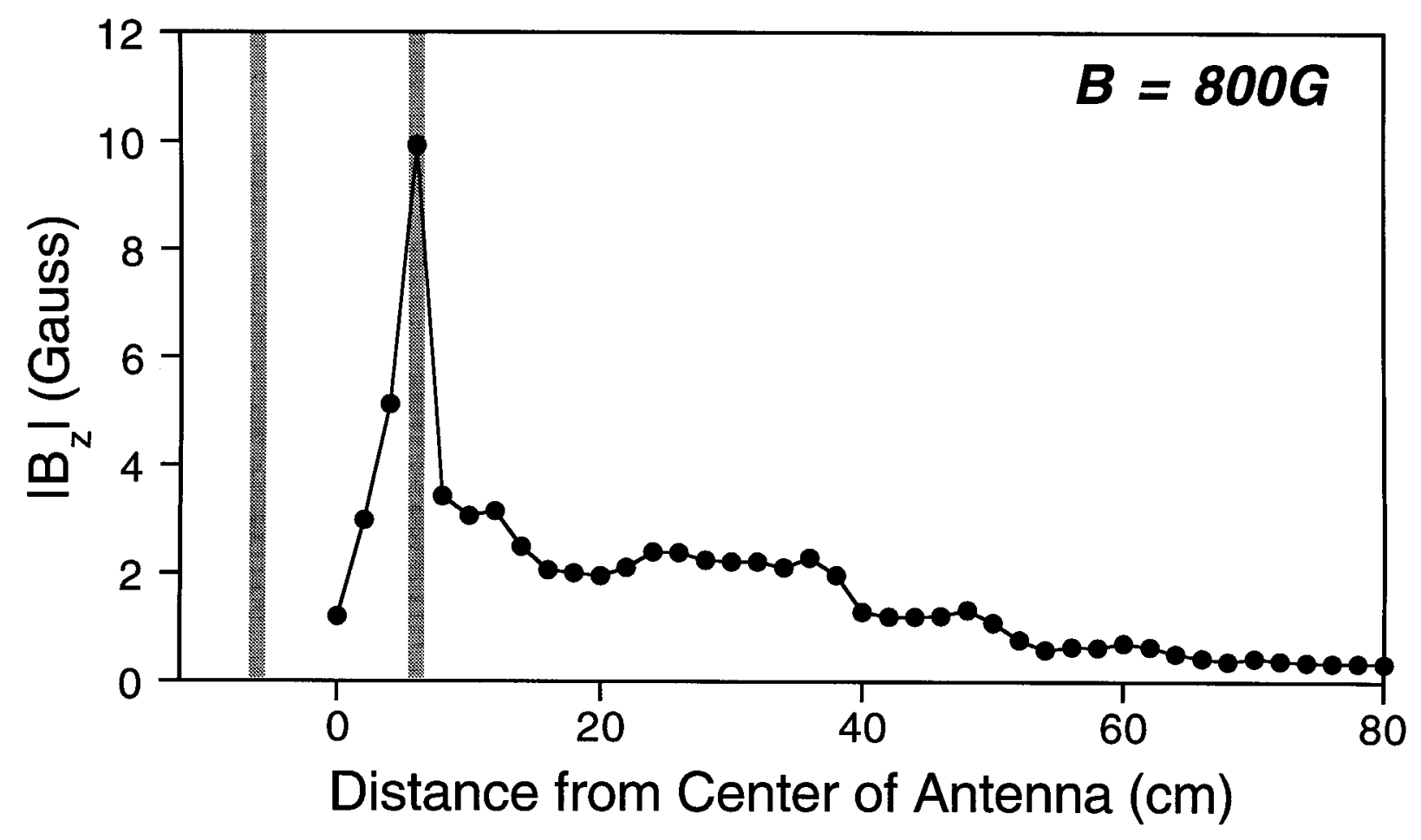

Fig. $6 e$ 


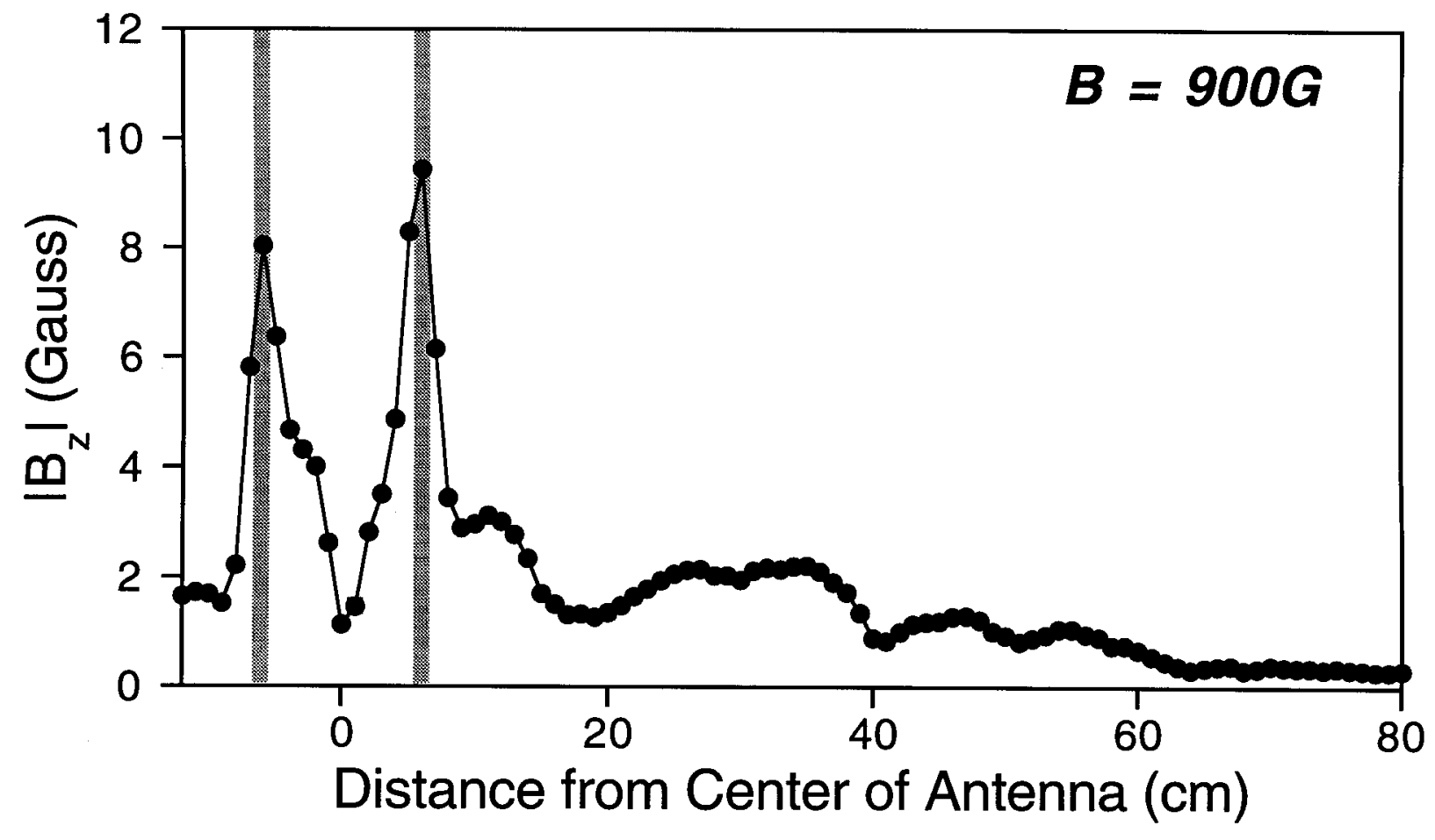

Fig. $6 f$ 


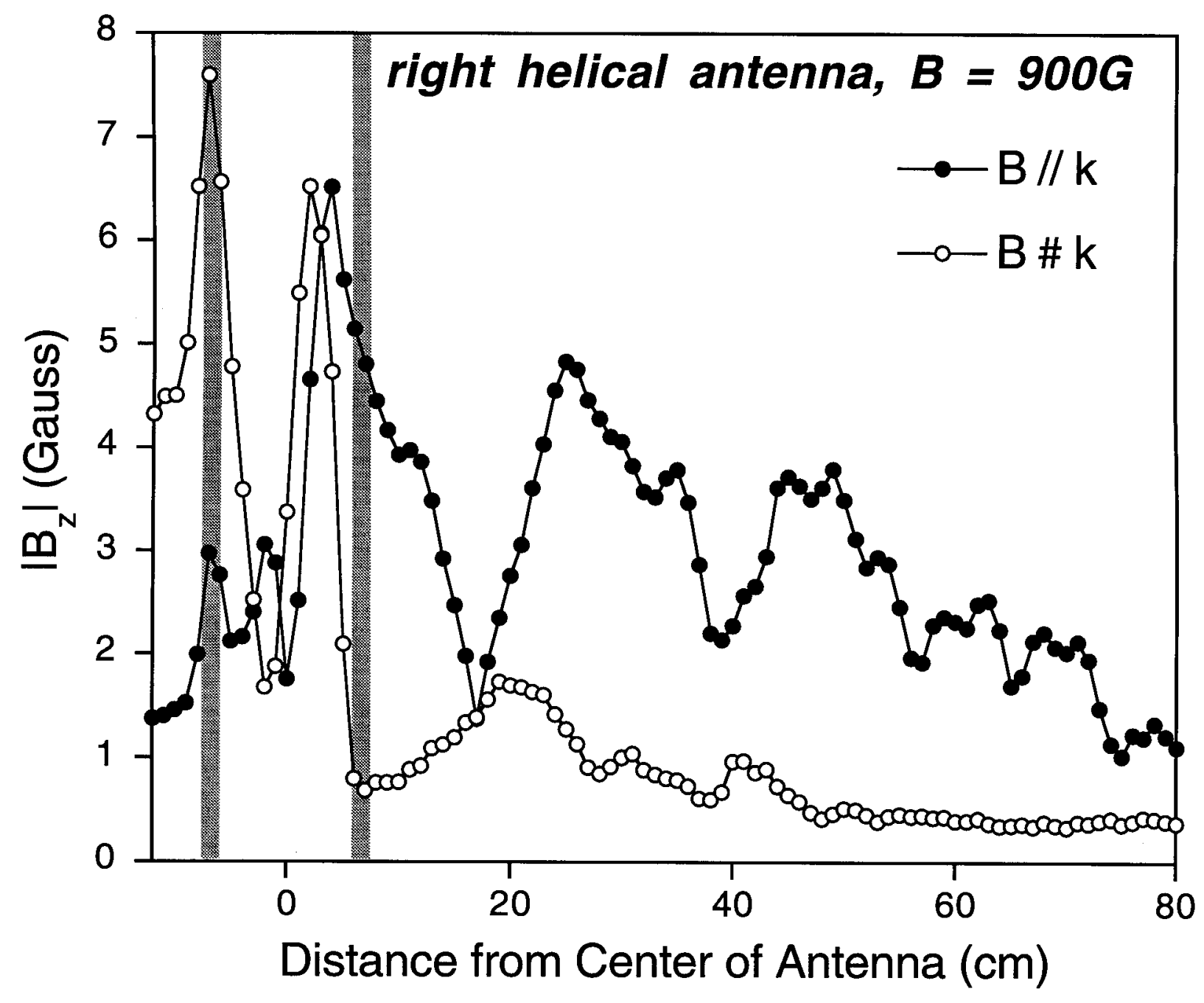

Fig. 7a 


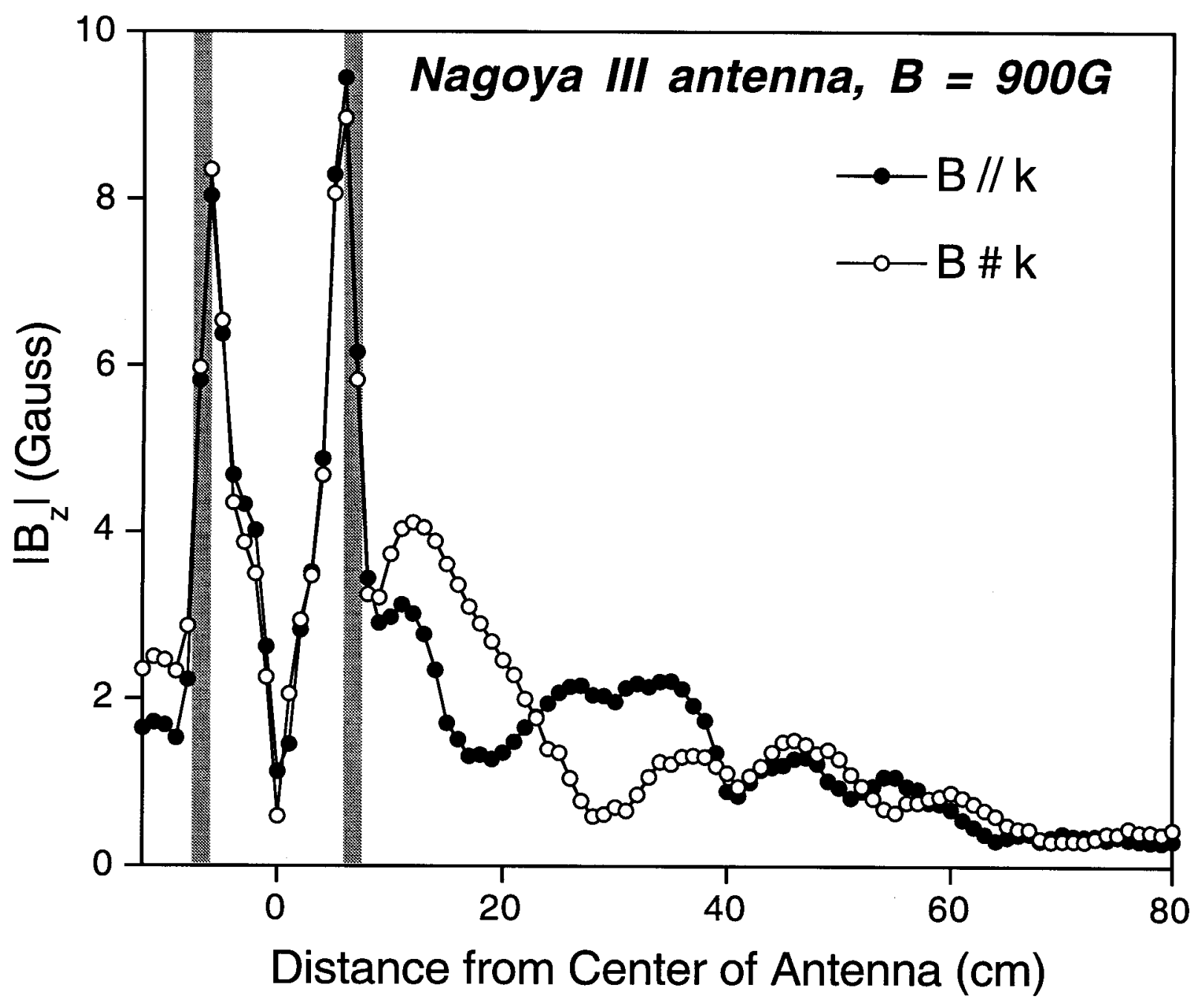

Fig. $7 b$ 


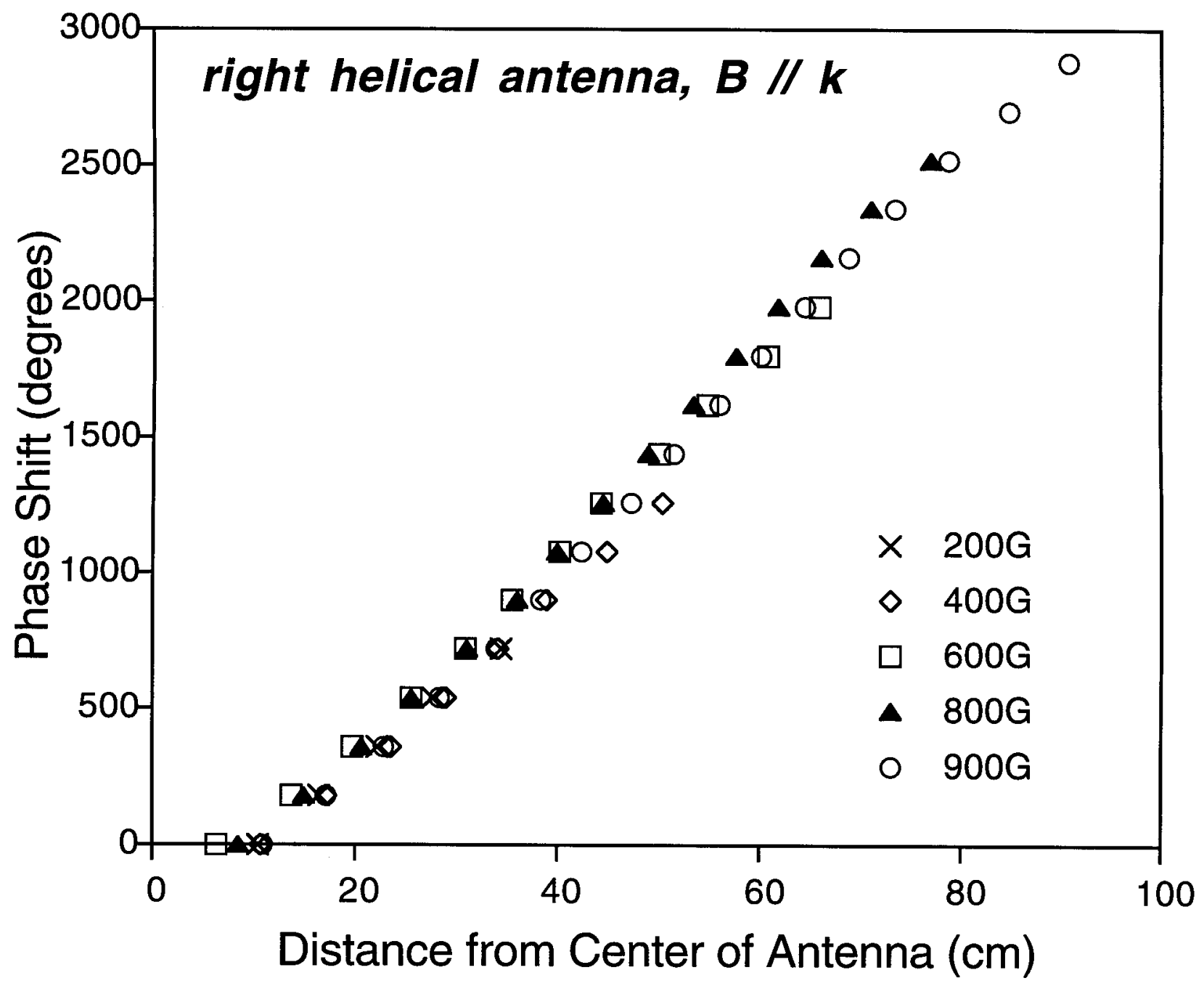

Fig. 8a 


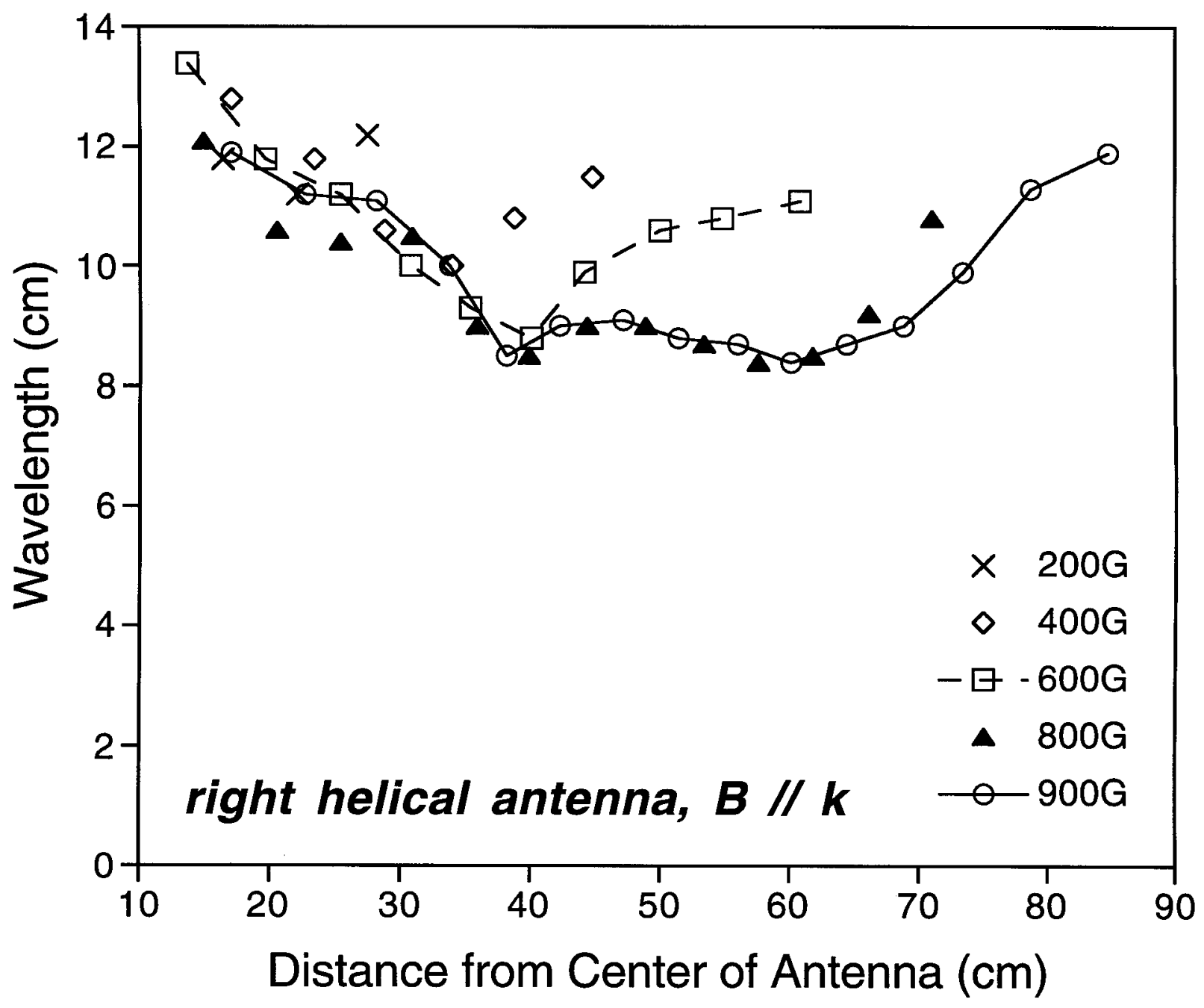

Fig. $8 b$ 


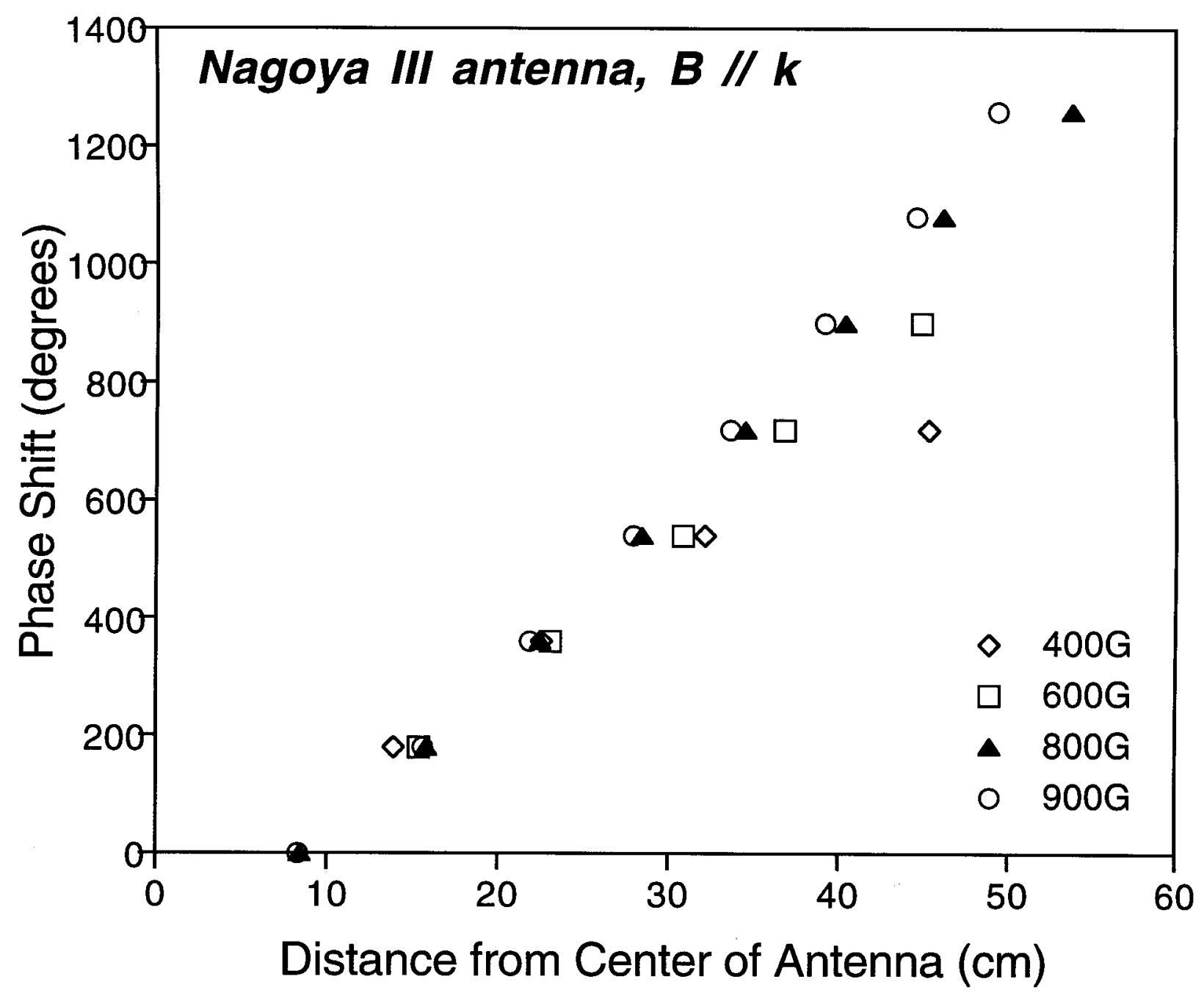

Fig. 9a 


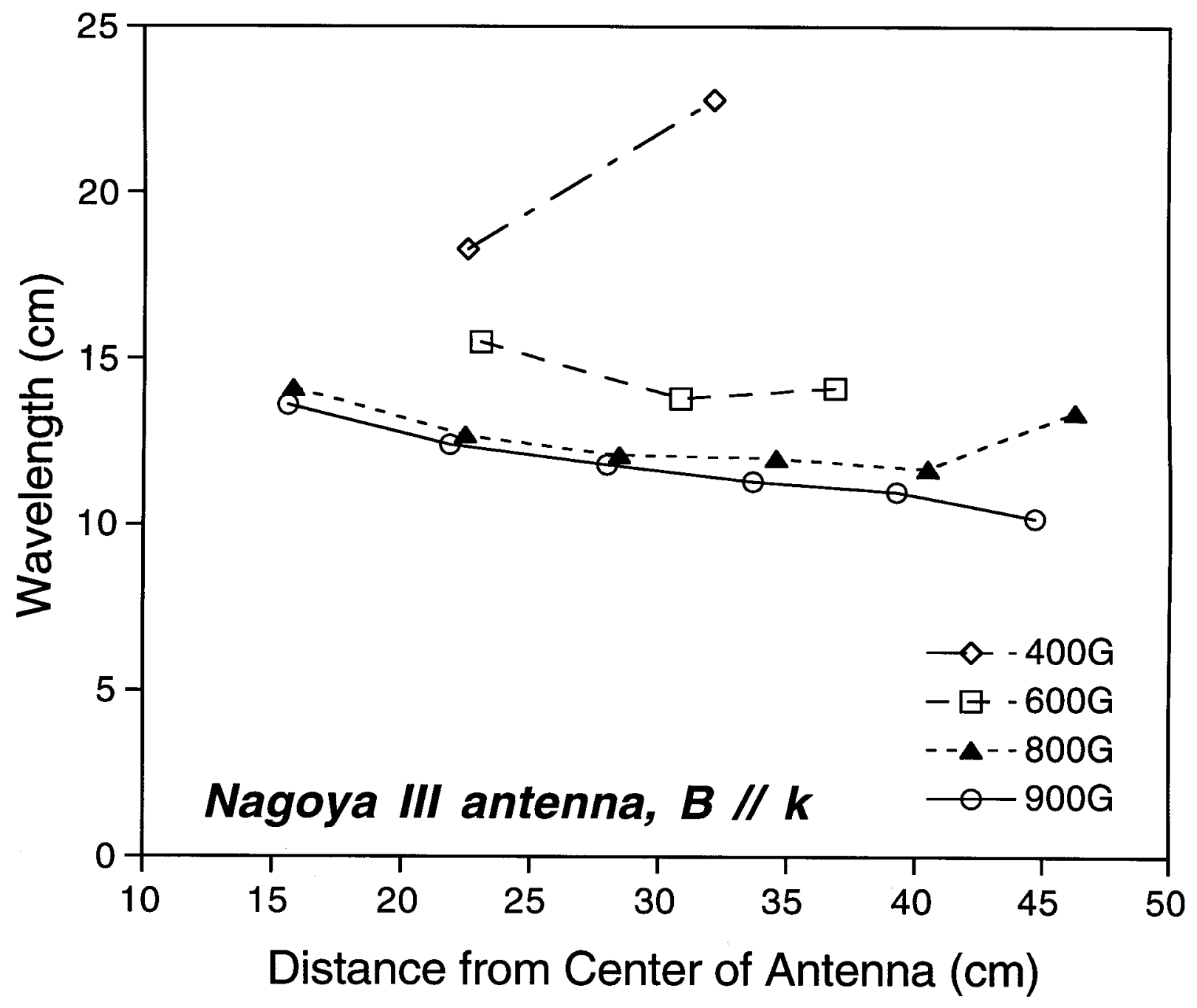

Fig. 9b 


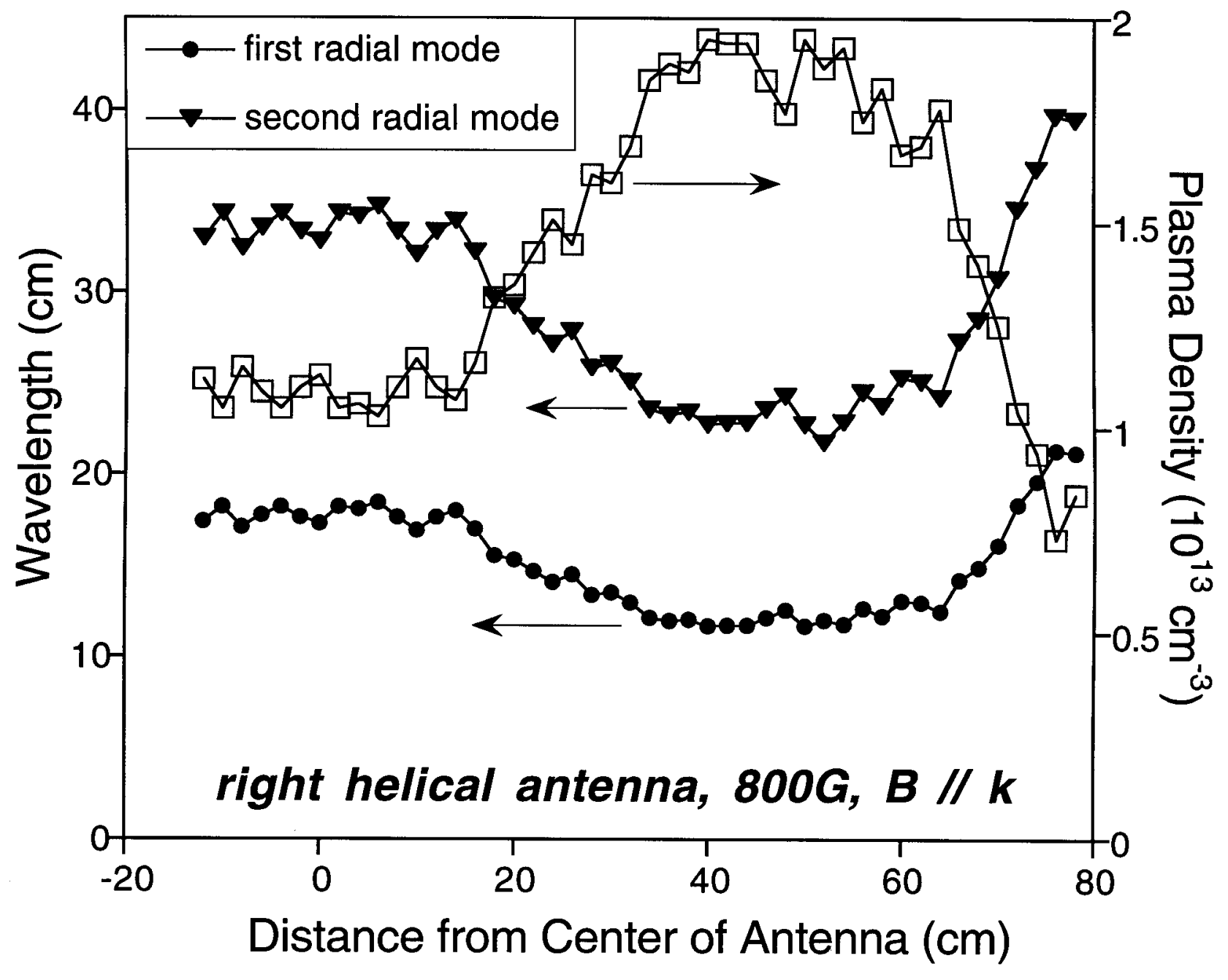

Fig. 10a 


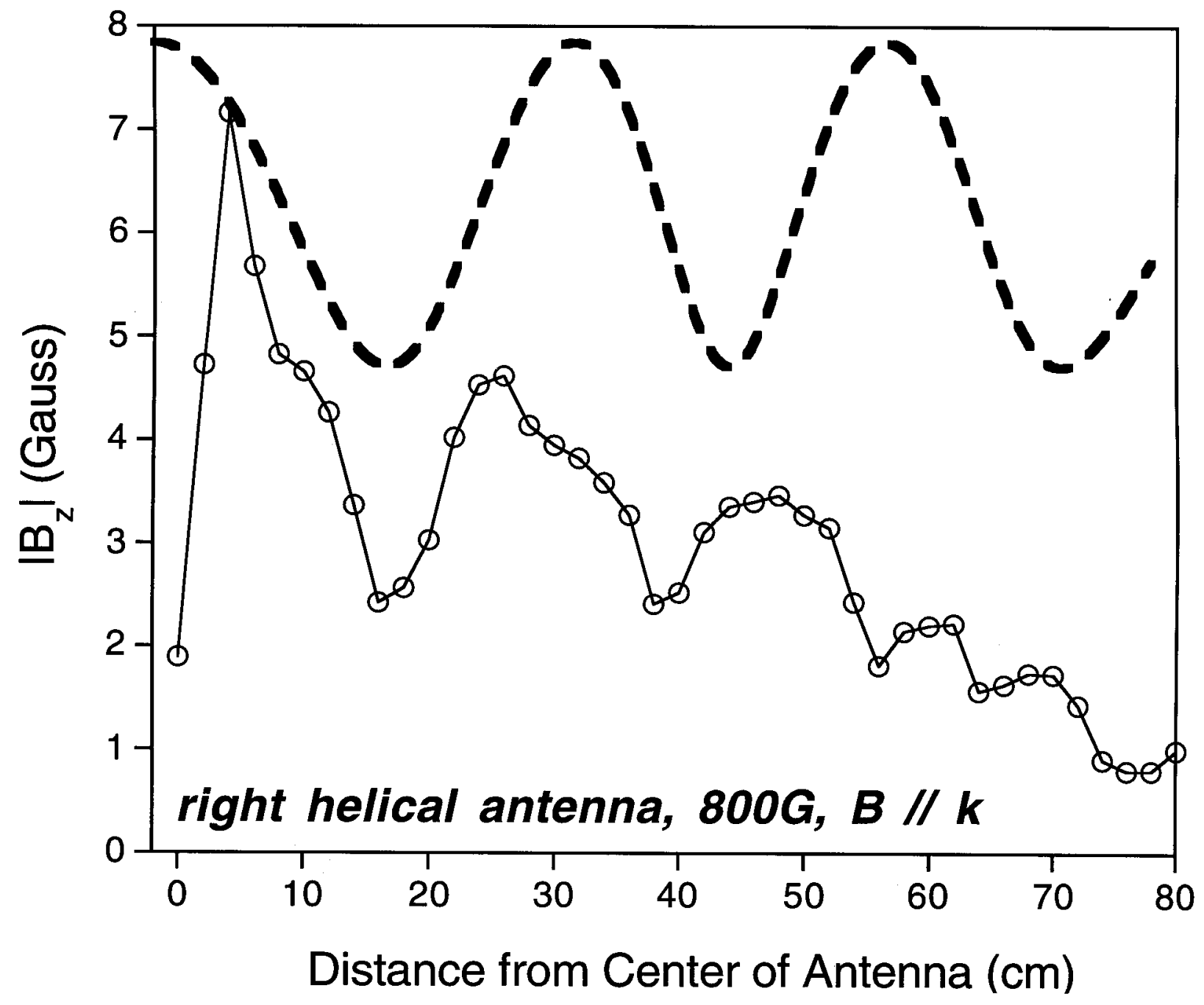

Fig. 11a 


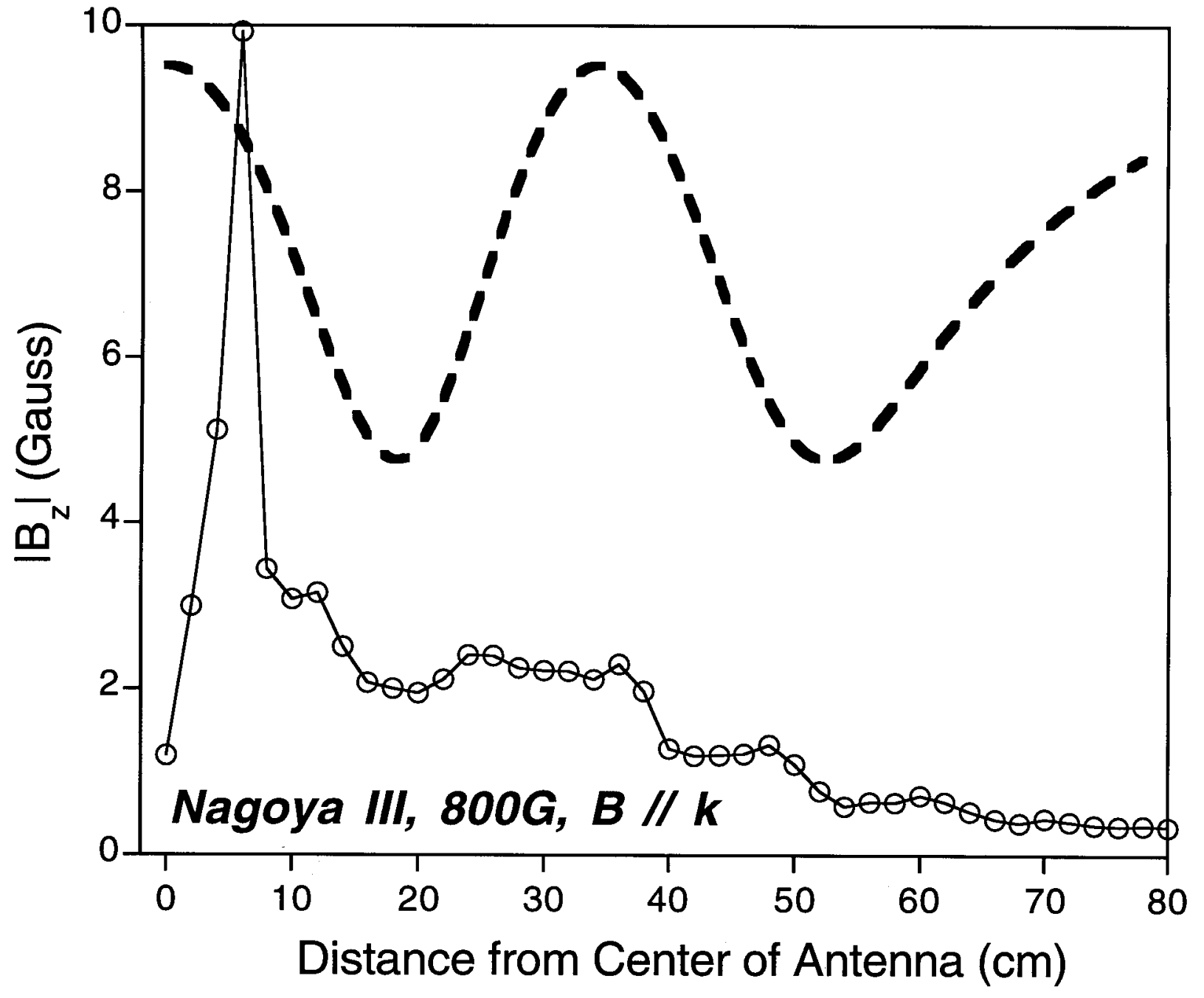

Fig. 11b 
k-Spectrum of Right-Helical Antenna

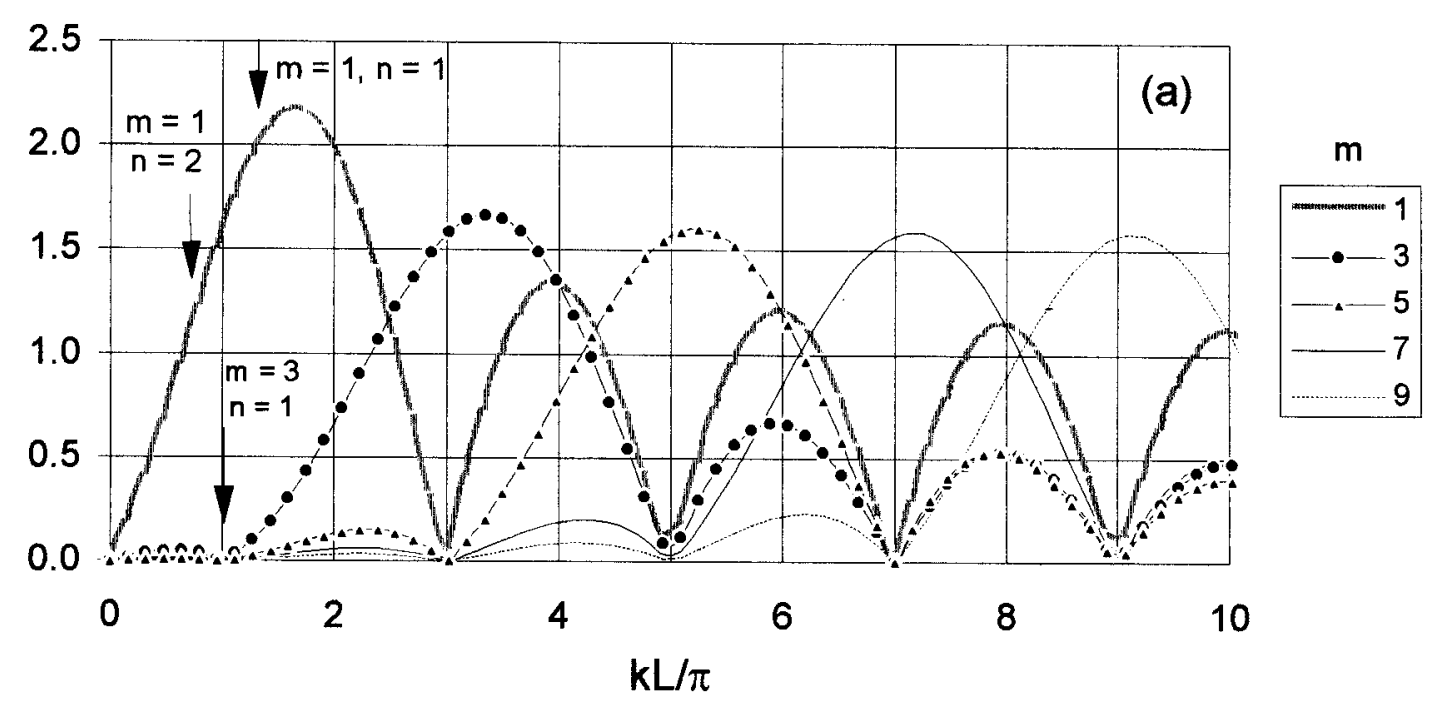

k-Spectrum of Nagoya III Antenna

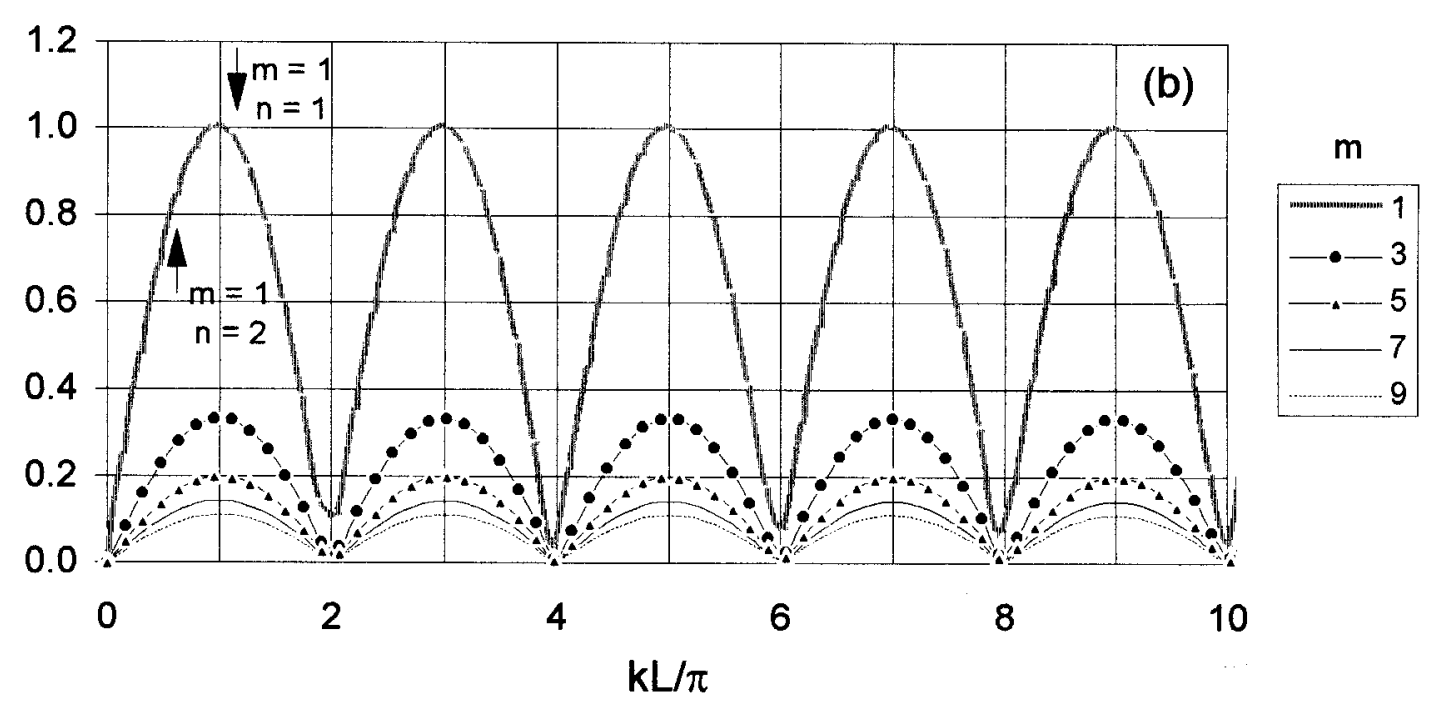

Fig. 12 


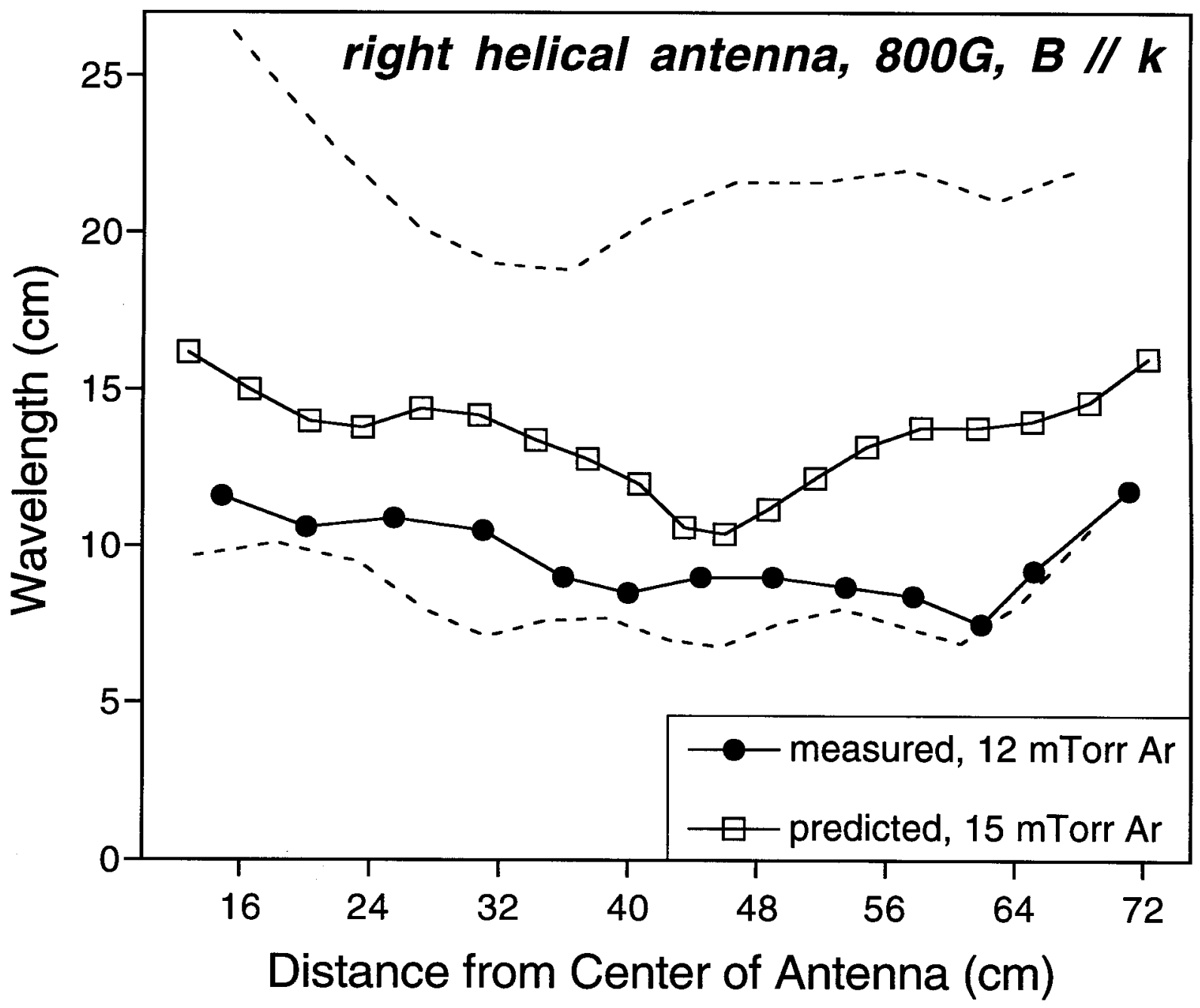

Fig. 13a 


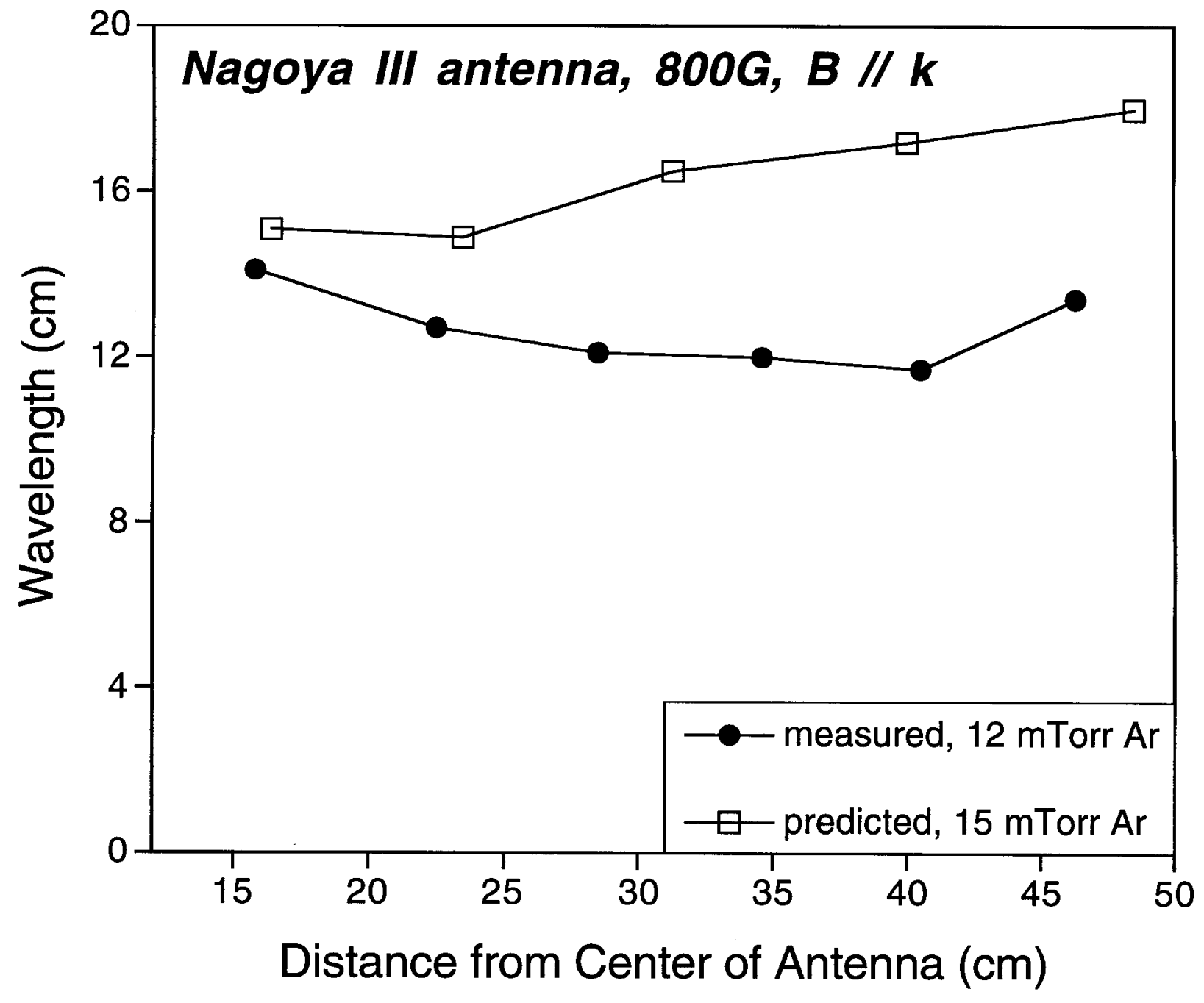

Fig. 13b 
Light and Chen, Phys. Plasmas 2, 4094 (1995)

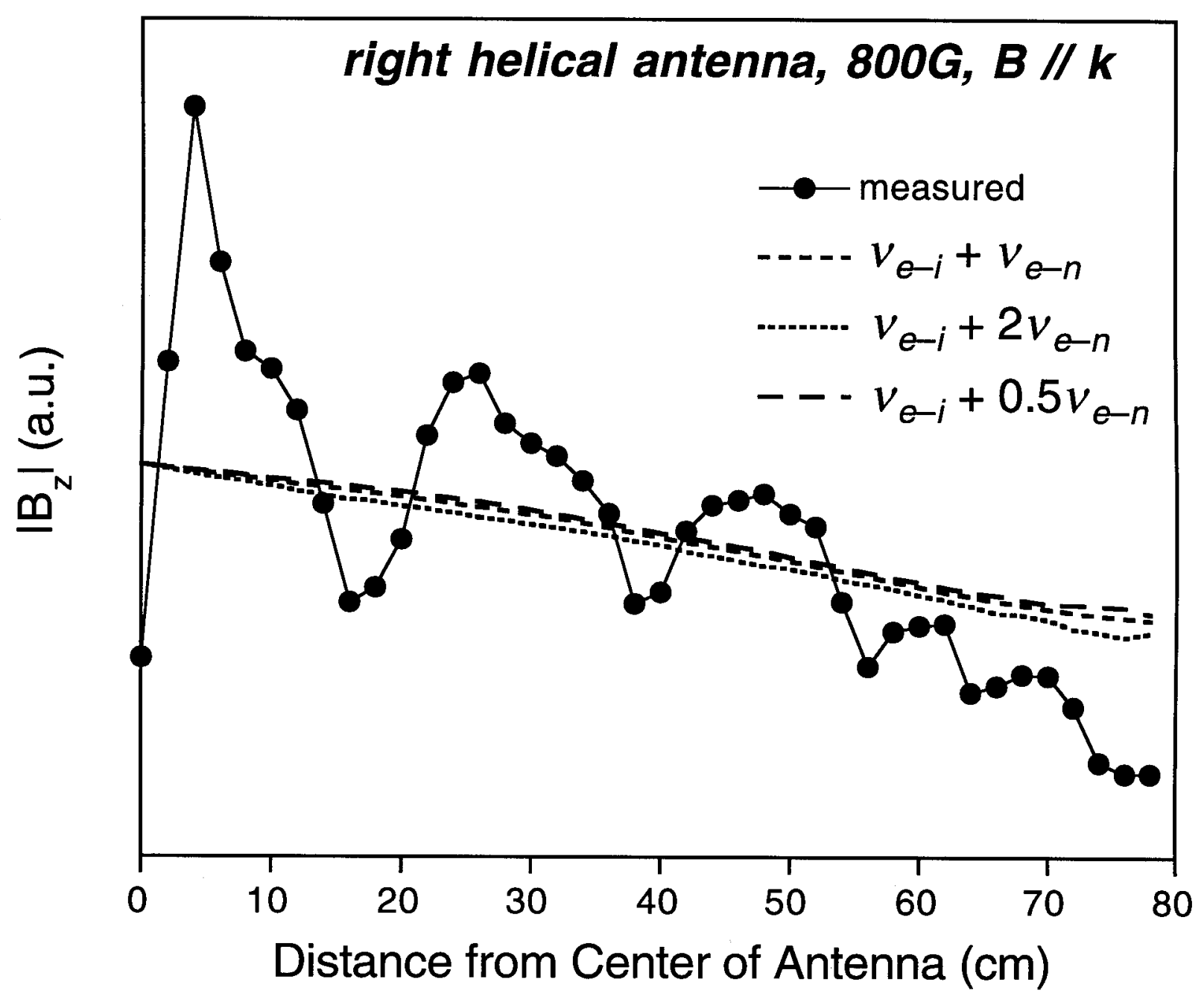

Fig. 14 